\title{
Dynamical flow characterization of transitional and chaotic regimes in converging-diverging channels
}

\author{
By A. M. GUZMÁN ANd C. H. AMON \\ Department of Mechanical Engineering, Carnegie Mellon University, Pittsburgh, PA 15213, USA
}

(Received 14 October 1994 and in revised form 19 March 1996)

Numerical investigation of laminar, transitional and chaotic flows in convergingdiverging channels are performed by direct numerical simulations in the Reynolds number range $10<R e<850$. The temporal flow evolution and the onset of turbulence are investigated by combining classical fluid dynamics representations with dynamical system flow characterizations. Modern dynamical system techniques such as timedelay reconstructions of pseudophase spaces, autocorrelation functions, fractal dimensions and Eulerian Lyapunov exponents are used for the dynamical flow characterization of laminar, transitional and chaotic flow regimes. As a consequence of these flow characterizations, it is verified that the transitional flow evolves through intermediate states of periodicity, two-frequency quasi-periodicity, frequency-locking periodicity, and multiple-frequency quasi-periodicity before reaching a non-periodic unpredictable behaviour corresponding to low-dimensional deterministic chaos.

Qualitative and quantitative differences in Eulerian dynamical flow parameters are identified to determine the predictability of transitional flows and to characterize chaotic, weak turbulent flows in converging-diverging channels. Autocorrelation functions, pseudophase space representations and Poincaré maps are used for the qualitative identification of chaotic flows, assertion of their unpredictable nature, and recognition of the topological structure of the attractors for different flow regimes. The predictability of transitional flows is determined by analysing the autocorrelation functions and by representing their attractors in the reconstructed pseudophase spaces. The transitional flow behaviour is examined by the geometric visualization of the evolution of the attractors and Poincaré maps until the appearance of a strange attractor at the onset of chaos. Eulerian Lyapunov exponents and fractal dimensions are quantitative parameters to establish the onset of chaos, the persistence of chaotic flow behaviour, and the long-term persistent unpredictability of chaotic Eulerian flow regimes. Lastly, three-dimensional simulations for converging-diverging channel flow are performed to determine the effect of the spanwise direction on the route of transition to chaos.

\section{Introduction}

Chaos theory has been applied to improve the understanding and exploitation of fluid mixing (Ottino 1992) and has been combined with classical fluid mechanics, transport phenomenon and kinematics to produce a general framework to investigate practical device performance in biomedical applications and heat exchangers (Hattler et al. 1992; Guzmán \& Amon 1994a). The objective in these investigations is the enhancement of mass and heat transfer involving transport processes near nonhomogeneous walls. Heat transfer enhancement due to fluid mixing exhibits different characteristics depending on the flow conditions. In a flow regime of poor mixing at 
low Reynolds numbers, trajectories of neighbouring fluid particles remain close to each other, and the convective mixing process is said to be non-chaotic. Increases in Reynolds numbers can lead to mixing enhancement owing to stretching and folding of fluid particles in flow regimes of chaotic advection (Ottino et al. 1992). Lastly, high heat transfer rates are obtained in flow regimes of turbulent mixing. However, larger mechanical pumping power is often required to induce heat and mass transfer enhancement by convective mixing at a given flow rate (Amon \& Mikic 1990). The question remains whether the advantage gained by increasing convective mixing is larger than the cost involved with the higher power input requirement.

In this context, there are two main reasons for our interest in characterizing and exploiting chaos. First, the behaviour of a chaotic system is a collection of many orderly behaviours, none of which dominates under ordinary circumstances. However, a chaotic system can follow one of its many regular behaviours when it is perturbed properly. In addition, chaotic systems are unusually flexible because of their ability to rapidly switch among different behaviours. Secondly, although chaos is unpredictable, it is deterministic. Therefore, if two nearly identical chaotic systems of the appropriate type are driven by the same signal, they will produce the same output, even though we cannot say a priori what the output might be (Douglas 1992; Ditto \& Pecora 1993; Shinbrot et al. 1993). Small changes in initial conditions can lead to large and unpredictable changes in the long-time evolution of the system. Therefore, no longrange behaviours can be anticipated. This also implies that computations of long-range behaviours can be seriously affected by small approximation errors.

Even though we are far from a complete understanding of all possible scenarios to chaos, three scenarios that lead to non-trivial attractors have been investigated theoretically and verified experimentally. These scenarios are the Ruelle-TakensNewhouse scenario through quasi-periodicity (Ruelle \& Takens 1971), the Feigenbaum scenario through period-doubling (Feigenbaum 1980), and the Manneville-Pomeau scenario through intermittency (Manneville \& Pomeau 1980).

In the Ruelle-Takens-Newhouse scenario, a system initially in steady state becomes dynamically unstable after changing its control parameter. As the motion grows and nonlinearities come into effect, the motion approaches a limit cycle through a Hopf bifurcation. As the control parameter is further varied, the system undergoes two or more Hopf bifurcations so that three simultaneously coupled limit cycles coexist, and chaotic motions then become possible. Thus, the precursor to such a chaotic state is a quasi-periodic multi-frequency state.

The classical Taylor-Couette flow experiment of Gollub \& Swinney (1975), using spectral analysis of laser Doppler velocimetry measurements, provided a remarkable verification of the Ruelle-Takens-Newhouse predictions. They observed a well-defined transition between a quasi-periodic two-frequency flow and a continuous frequency spectrum flow. Quasi-periodic Taylor-Couette flows with two and three independent frequencies as well as non-periodic flow behaviour corresponding to low-dimensional deterministic chaos were both observed experimentally (Gorman, Reith \& Swinney 1980; Brandstater \& Swinney 1987) and predicted numerically (Vastano \& Moser 1991). Pfister, Buzug \& Enge (1992) also identified different routes to chaos in Taylor-Couette flows and found a period-doubling cascade, a quasi-periodic threefrequency flow, and an intermittent scenario.

Gollub \& Benson (1980) identified four different routes to turbulence in Rayleigh-Bénard convective flows depending on the geometric aspect ratio, Prandtl number, and mean flow conditions. They found broad-spectrum flows preceded in Rayleigh number by quasi-periodicity and phase locking, by quasi-periodic two- and 
three-frequency flows, by a succession of subharmonic period-doubling bifurcations, and by intermittent noise. Walden et al. (1984) observed experimentally quasi-periodic flows in Rayleigh-Bénard convection with four and five independent frequencies, whereas McLaughlin \& Orszag (1982) found numerically periodic, quasi-periodic, and chaotic behaviour in their investigation on transitional thermal convection. Their results appear to be consistent with the Ruelle-Takens-Newhouse theory in that simulated flows with three or more distinct frequencies also contain broadband frequency components.

Guzmán \& Amon (1994a) demonstrated that a two-dimensional flow in a converging-diverging channel experiences a sequence of instabilities reaching a chaotic regime after three distinct supercritical Hopf bifurcations as the Reynolds number is increased. As a consequence of classical fluid dynamics flow characterizations, such as velocity histories and power spectra, we found that this converging-diverging channel flow bifurcates successively from a laminar steady state to an aperiodic regime, passing through intermediate states of periodicity with one fundamental frequency, twofrequency quasi-periodicity, frequency-locking periodicity, and three-frequency quasiperiodicity. When a system undergoes three Hopf bifurcations, starting from a stationary solution as its control parameter is varied (e.g. Reynolds number), then, it is likely that the system possesses a strange attractor with sensitivity to initial conditions after the third bifurcation (Ruelle 1980; Eckmann 1981). The power spectrum of such a system exhibits one, then two, and possibly three independent fundamental frequencies. As the third frequency is about to appear, some broadband components will appear simultaneously if there is a strange attractor. This succession can be interpreted as a chaotic evolution of the system governed by a strange attractor which culminates with a continuous frequency spectrum solution that emerges after a quasi-periodic flow, suggesting that the transition to turbulence follows the Ruelle-Takens-Newhouse (RNT) scenario (Eckmann 1981; Guzmán \& Amon 1994a).

In this paper, we extend our previous investigations in converging-diverging channels by performing direct numerical simulations (DNS) to calculate dynamical system parameters, which provide strong evidence of the Ruelle-Takens-Newhouse route to chaos. We employ dynamical system techniques, such as time-delay reconstructions of pseudophase spaces, Poincaré maps, autocorrelation functions, fractal dimensions, and Eulerian Lyapunov exponents to characterize laminar, transitional and chaotic flow regimes. In addition, we examine qualitative and quantitative differences in these dynamical system parameters to identify Reynolds number ranges of chaotic flow behaviour and to quantify the strength of chaotic regimes so that chaotic mixing can be conveniently exploited.

This paper is organized as follows. In $\$ 2$, we describe the physical problem, the mathematical model, and the numerical procedure. In $\S 3$, we present the numerical algorithms for evaluating dynamical system parameters, we analyse the Eulerian flow dynamical representation, and we describe the route to chaos using techniques derived from dynamical systems. In $\$ 4$, we present Lagrangian trajectories on laminar and transitional flow regimes, and in $\$ 5$, we describe the effects of flow three-dimensionality. In $\$ 6$, we discuss the relevance of the dynamical parameter characterization for investigating transition to turbulence and chaotic flow regimes, followed by a discussion of our current and future research. Lastly, conclusions are presented in $\S 7$. 


\section{Converging-diverging channel: physical problem, mathematical model and numerical approach}

The converging-diverging channel is a symmetric, wavy channel with sinusoidal walls (figure 1). It is a model for devices employed for enhancing heat and mass transfer efficiency in industrial transport processes and in biomedical applications such as blood membrane oxygenators and kidney dialysers. Numerical calculations and experimental studies have been performed in converging-diverging channels and global flow patterns have been reported for fully-developed steady flows and pulsating external flows (Sparrow \& Prata 1983; Sobey 1985; Ralph 1986; Faghri \& Asako 1987; Nishimura et al. 1990; Guzmán \& Amon 1993). These flows are characterized by a separation zone for low Reynolds numbers, and by a steady vortex regime for moderate Reynolds numbers with recirculation zones and mixing regions within the mainstream. As the Reynolds number increases, unsteady vortex motion develops leading to the onset of transitional flows.

We reported the basic mechanisms of unsteady oscillatory motion in convergingdiverging channels and obtained symmetric vortical structures for subcritical flow regimes and asymmetric, time-dependent, self-sustained oscillatory flows for supercritical regimes (Guzmán \& Amon 1994a). We demonstrated that the vortex dynamics is synchronized with the travelling wave structure which corresponds to Tollmien-Schlichting (TS) waves. Furthermore, the instabilities responsible for inducing and sustaining supercritical oscillatory flows are TS instabilities, and the oscillatory flow frequency corresponds to the frequency associated with the least-stable TS channel mode.

Heat transfer enhancement is achieved in self-sustained oscillatory flow regimes with minimum penalties associated with increases in viscous dissipation and pumping power (Amon \& Mikic 1990; Greiner, Chen \& Wirtz 1991). Such enhancement in heat transfer is related to the flow mixing that occurs in self-sustained oscillatory flows (Amon et al. 1992), while mixing is related to the stretching and folding of fluid elements, and stretching and folding constitute the fingerprint of chaos (Sobey 1985; Ottino et al. 1992).

The flow in the converging-diverging channel, shown in figure 1 , is periodically fully developed in the streamwise $x$-direction, homogeneous in the spanwise $z$-direction, and governed by the incompressible Navier-Stokes and continuity equations in the computational domain $\Omega$ defined by the periodicity length $L$. The non-dimensionalized lengths, $L / h=9.33, H / h=6.66$, and $W / h=53.33$, are associated with the $x$-, $y$ - and $z$-directions, respectively. The boundary conditions for the velocity are no slip at the sinusoidal walls, periodicity on $\partial \Omega_{p}$ of the fully-developed flow in the $x$-direction, and no slip at the sidewalls of the channel. The Reynolds number is defined as $R e=\frac{3}{2} V h / \nu$, where $V$ is the time-mean cross-channel average velocity, $h$ is the channel half-height, and $\nu$ is the kinematic viscosity of the fluid. All variables are non-dimensionalized by the centreline velocity $\frac{3}{2} V$, the channel half-height $h$, and the convective time.

To establish the adequacy of the periodic boundary conditions imposed, our numerical results were compared with experimental flow visualizations in the available Reynolds number range $5<R e<300$ (Stephanoff, Sobey \& Bellhouse 1980; Sobey 1980, 1982; Nishimura et al. 1990) and with numerical predictions from a computational domain composed by different periodicity lengths (Guzmán \& Amon $1994 b$ ). These comparisons are in good agreement for the range of Reynolds number explored, validating the computational domain utilized for the simulations reported in this paper. 


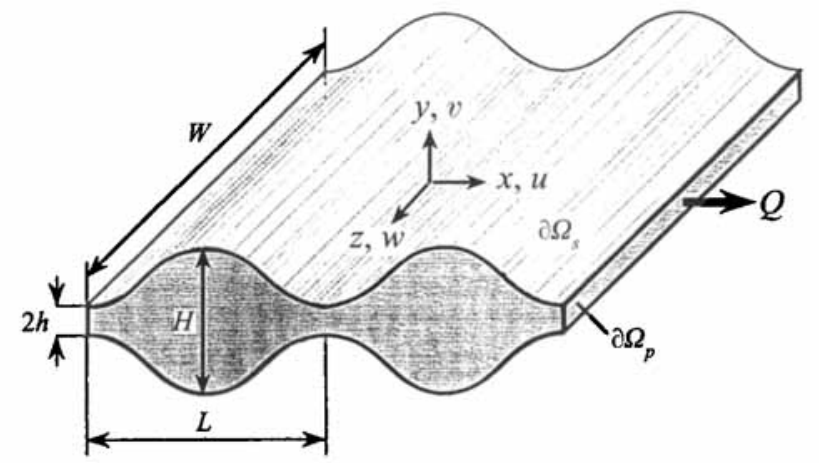

FIGURE 1. Converging-diverging channel geometry: non-dimensionalized periodicity length $L / h=9.33$, height $H / h=6.66$, and spanwise length $W / h=53.33$. Point coordinates $=$ $(x / h, y / h, z / h): P_{1}=(4.67,3.33,-24.0) ; P_{2}=(4.67,3.33,0.0) ; P_{3}=(4.67,3.33,24.0)$.

The Navier-Stokes and continuity equations with appropriate boundary conditions are solved numerically using the spectral element method with a three-step timesplitting scheme (Patera 1984; Amon 1993, 1995). This time-stepping procedure includes an explicit third-order forward-in-time Adams-Bashforth scheme for the nonlinear convective term, an implicit Euler-backward scheme for the pressure step, and an implicit Crank-Nicolson scheme for the viscous step. The time-step size through the Courant number condition, the enforcement of the incompressibility constraint, and the viscous corrections along with the boundary conditions are imposed at the nonlinear, pressure and viscous steps, respectively. In the spectral element spatial discretization, the domain is divided into macro-elements which are isoparametrically mapped from the physical space to the local coordinate system. The geometry, velocity and pressure in each macro-element are represented as a tensor product of high-order Lagrangian interpolants through Gauss-Lobatto-Chebyshev collocation points (Amon 1993). The nonlinear convective term is evaluated pseudospectrally whereas the pressure and viscous terms, which correspond to modified Helmholtz equations, are solved by a variational approach (Patera 1984). This non-diffusive numerical methodology is well-suited to investigate temporal transition in self-sustained oscillatory flows since high-frequency flow oscillations are not damped by artificial numerical viscosity. Moreover, since the predicted flows are the results of evolution calculations, they correspond to stable solutions that are physically realizable.

For the three-dimensional simulations, the computational domain is discretized with 10 layers of macro-elements in the spanwise $z$-direction with 125 nodes per macroelement (figure 2). Each layer contains 32 macro-elements with the same $(x, y)$-plane discretization as the one used for the two-dimensional simulations. Figure $2(a)$ shows the macro-elements in the discretized domain whereas figures $2(b)$ and $2(c)$ depict twodimensional projections of the spectral element mesh. The dark lines correspond to the boundaries between spectral macro-elements and the light lines are the internal collocation mesh points. Computations were performed with different mesh resolutions to determine the adequacy of the spatial discretization to resolve all temporal and spatial scales of the different flow regimes investigated (Guzmán \& Amon 1994a).

The numerical results are obtained by direct simulation of the time-dependent governing equations, integrating in time, starting with a predicted flow and increasing gradually the Reynolds number until a steady, time-periodic or transitional state is found. Increases in Reynolds numbers are made in relatively small steps, so that the 

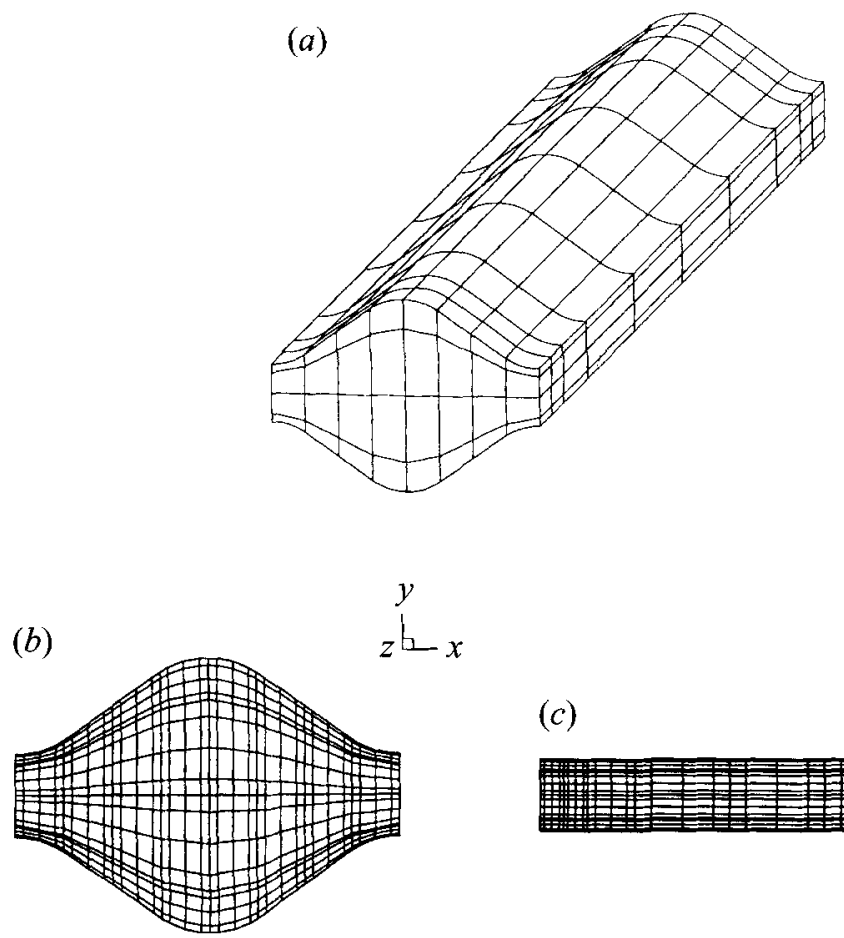

(c)

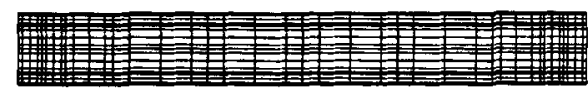

FIGURE 2. Computational domain and typical spectral element mesh: (a) three-dimensional macroelement discretization. Two-dimensional mesh projection depicting internal nodes; $(b) z$-axis view; (c) $y$-axis view.

dynamic flow evolution can be followed in detail. The initial conditions for subsequent simulations are those associated with the preceding lower Reynolds number. To investigate the possibility of hysteresis effects in transitional predictable flows, several cases were simulated using different initial conditions corresponding to flow fields at both lower and higher Reynolds numbers. For example, simulations were performed for $R e=400$ using as initial conditions the flow fields corresponding to $\operatorname{Re}=150,300$ and 500. These simulations yielded the same time-asymptotic evolution of the flow pattern and the same oscillatory frequencies, which suggest that the solutions are independent on the initial conditions for Reynolds numbers up to 550. After initial transients in the time evolution disappear, simulations are performed for an integration time long enough to yield statistically stationary signals to within $0.5 \%$. This requires about $10^{5}$ timesteps for a Reynolds number of 750 .

For subcritical Reynolds numbers, $R e<R e_{c} \approx 130$, the flow patterns are symmetric along the centreline of the converging-diverging channel, and no flow oscillations are present. These time-asymptotic states, resulting from transient flow evolutions, are characterized by separation zones and symmetric vortices. For supercritical Reynolds numbers, $R e>R e_{c}$, the flow field loses its symmetry, and self-sustained oscillatory flows are induced which evolve to states of periodicity, quasi-periodicity and aperiodicity through successive flow bifurcations. These flows become more complex with larger oscillatory amplitudes and smaller multi-frequencies as the Reynolds number is increased.

We plot in figure 3 the periodically fully-developed streamlines predicted numerically for subcritical and supercritical Reynolds number flows. Figure $3(a)$ shows symmetric streamlines obtained for $R e=10$. There is no flow separation along the sinusoidal 

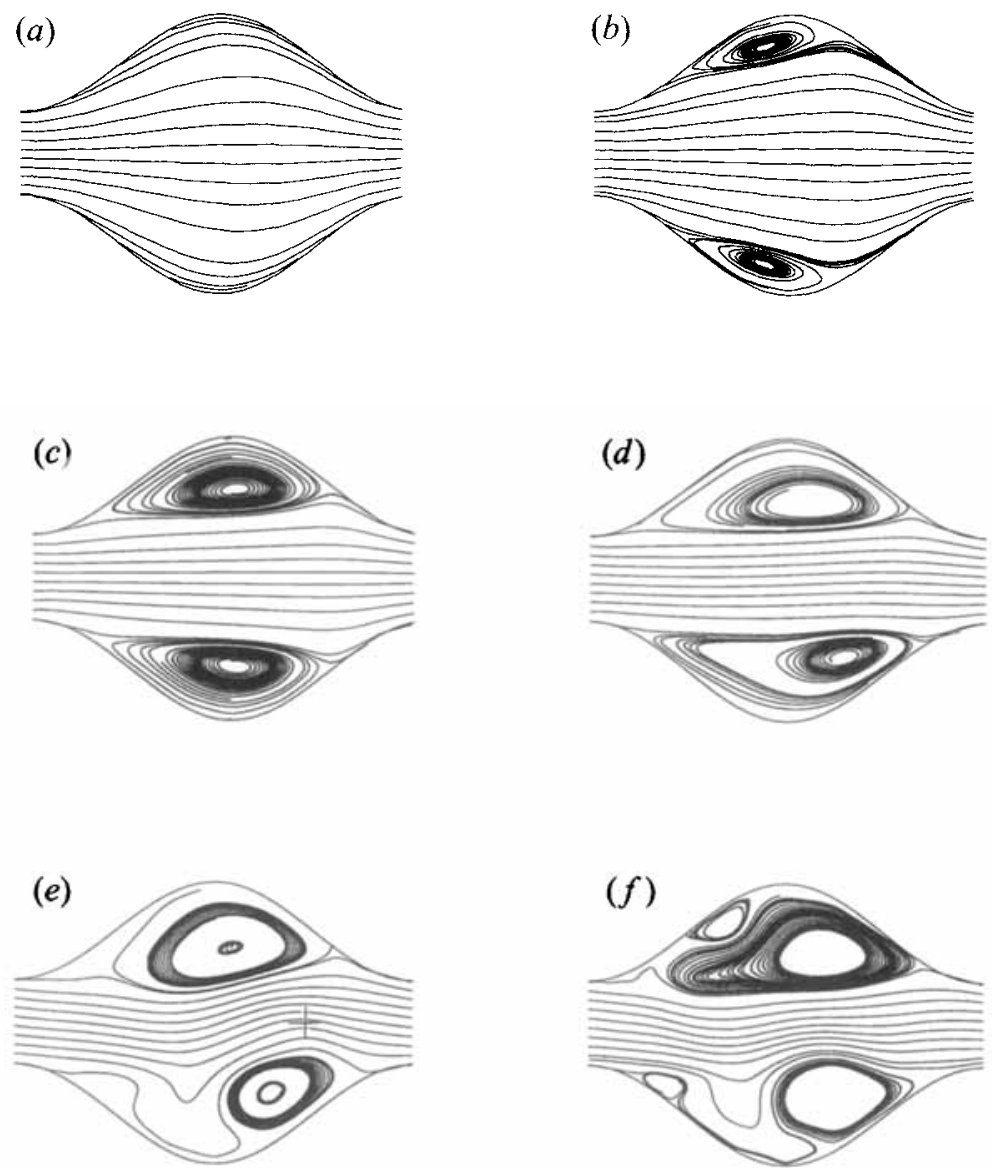

FIGURE 3. Instantaneous streamlines for subcritical and supercritical Reynolds flow regimes:

(a) $R e=10 ;(b) R e=20 ;(c) R e=50 ;(d) R e=150 ;(e) R e=400 ;(f) \operatorname{Re}=750$.

walls and no vortex formation in this flow regime which corroborates the experimental flows visualized by Nishimura et al. (1990). Figure $3(b)$ depicts symmetric counterrotating vortices located upstream in the furrows and close to the walls for $R e=20$. These vortices become stronger, increasing in size and moving downstream for $R e=50$ as shown in figure $3(\mathrm{c})$. Further downstream motions of the vortices occur as the Reynolds number is increased. These flow patterns agree well with those previously obtained experimentally and numerically for steady-state conditions (Sobey 1980, 1982; Nishimura 1990). As the flow bifurcates to a self-sustained oscillatory flow, the vortex dynamics depicts an asymmetric motion of the vortices in the furrows and an increasing-decreasing-bulging-ejection vortical sequence for higher Reynolds numbers. Figure $3(d)$ shows instantaneous streamlines for $R e=150$. In this two-vortex flow regime, the recirculating regions are moving alternately in the upper and lower furrows shifting their positions and strengths; however, the vortices remain confined in the furrows. At higher Reynolds numbers, the level of asymmetry and complexity of the flow structure increases as a consequence of a second flow bifurcation to a quasiperiodic regime (Guzmán \& Amon 1994a). A new flow regime is established and depicted in figure $3(e)$ by instantaneous streamlines at $R e=400$. The viscous forces are not strong enough to keep the vortices confined in the sinusoidal furrows and the 
vortices are alternatively ejected. The vortex dynamics is synchronized with the wavy character of the flow which is induced by the travelling waves and by the forces caused by the pressure field (Amon \& Mikic 1990). These forces drive the flow up and down in the furrows as the wavy flow convects downstream. For example, as the crest of the wave passes through the furrows, its relatively high-pressure region, indicated with + in figure 3(e), pushes fluid into the upper furrow while its relatively low-pressure region induces the vortex bulging from the lower furrow.

As the Reynolds number is increased further, the flow becomes aperiodic. The instantaneous streamlines for $R e=750$, shown in figure $3(f)$, depict the complexity of the flow structure and vortex interactions. The travelling wave structure and overall flow patterns remain; however, this flow presents smaller scale and stronger vortices than the preceding Reynolds number flows. Clockwise and counter-clockwise rotating vortices with different strengths co-exist in the same furrow, inducing stronger mixing between the bulk flow and the fluid in the furrows. On the basis of classical flow analyses, this aperiodic flow could be either chaotic or quasi-periodic with incommensurate fundamental frequencies and their harmonics. Therefore, our objective in this work is to combine DNS time-dependent flow solutions with nonlinear dynamical system techniques to characterize further these transitional flow regimes, to establish the evolutive pattern for the origin of chaos, to quantify chaotic flow regimes, and to provide evidence of the Ruelle-Takens-Newhouse (RTN) route for transition to turbulence in converging-diverging channel flows.

\section{Dynamical characterization of transitional and chaotic Eulerian flow regimes}

In this section, we present the dynamical characterization of Eulerian flows in converging-diverging channels in terms of autocorrelation functions, pseudophase space representations, Poincaré sections, Eulerian Lyapunov exponents, and fractal dimensions for periodic, quasi-periodic and chaotic flow regimes. The autocorrelation functions, pseudophase space representations, and Poincaré techniques are qualitative tools that allow us to assert the unpredictable nature of chaotic flows and, at the same time, to recognize the topology of the attractor for different flow regimes. The Eulerian Lyapunov exponents and fractal dimension techniques are quantitative tools to establish the onset of chaos, the persistence of chaotic flow regimes, and the long-term persistent unpredictability of chaotic flows.

\subsection{Autocorrelation functions}

The autocorrelation function, $A(T)$, is a useful measure of the predictability of the fluid motion. For a periodic signal, $A(T)$ is a periodic function, whereas, for a chaotic signal, $A(T)$ tends to zero for some $T>T_{c}$, where $T_{c}$ is a measure of the time during which the motion can be predicted in the future. $A(T)$ is defined as

$$
A(T)=\frac{1}{\left(t_{2}-t_{1}\right)} \int_{t_{1}}^{t_{2}} x(t) x(t+T) \mathrm{d} t,
$$

where $T$ is a delay time between $t_{1}$ and $t_{2}$.

Figure 4 shows the autocorrelation functions obtained from the temporal evolution of the streamwise $U$-velocity component for various Reynolds numbers at a typical point located at the channel centreline. We performed extensive simulations for long enough times to evaluate the autocorrelation function $A(T)$ as $T$ goes to infinity, and 
(a)

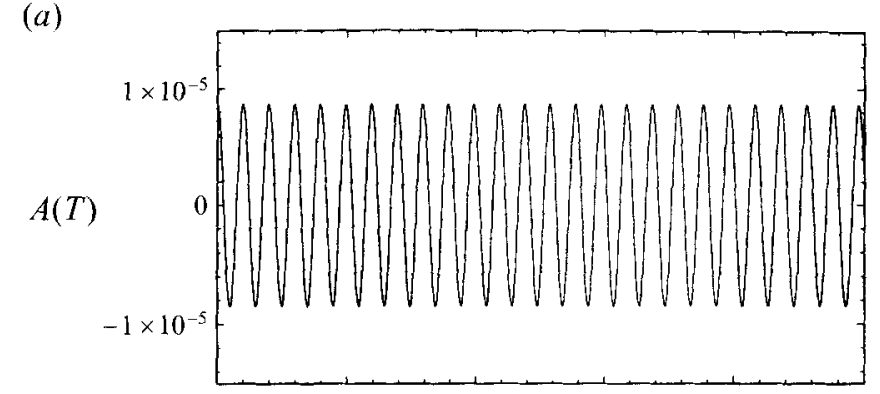

(b)

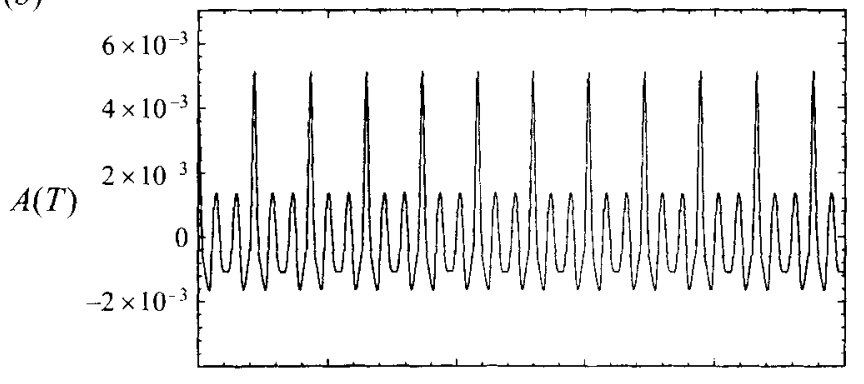

(c)

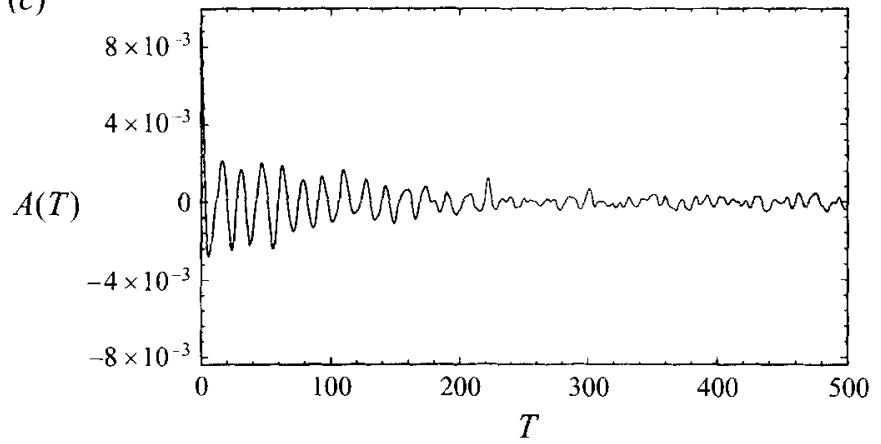

Figure 4. Autocorrelation functions $A(T)$ of the $U$-velocity: (a) $R e=150 ;(b) R e=400$; (c) $\operatorname{Re}=850$

we plot $A(T)$ corresponding to the last $T=500$ units of non-dimensional time. Figure 4 (a) depicts a periodic autocorrelation function generated by the temporal periodic flow evolution at $R e=150$. A similar periodic autocorrelation function is obtained at $R e=400$ corresponding to the frequency-locking flow regime (figure $4 b$ ), whereas for higher Reynolds numbers, the autocorrelation function tends to zero for sufficiently long times. This implies the existence of a chaotic flow regime and an unpredictable flow behaviour as illustrated in figure $4(c)$ for $R e=850$.

The frequency-locking phenomenon, also called quasi-periodicity in mode-locking, plays a crucial role in the chosen route to chaos. Depending on the extent of change in the first fundamental frequency, $\omega_{1}$, it has been found that the mode-locking regime may become locked in another frequency ratio (Bergé, Pomeau \& Vidal 1986; Moon 1992), or the motion may suddenly become chaotic as the control parameter increases (Gollub \& Benson 1980; Vittori \& Blondeaux 1993). In converging-diverging channel flows, we have found that the frequency-locking regime at $R e=400$ reaches an asymptotic plateau and, then, evolves to a quasi-periodic regime. As the Reynolds number is increased beyond the first flow bifurcation, a self-sustained oscillatory flow 


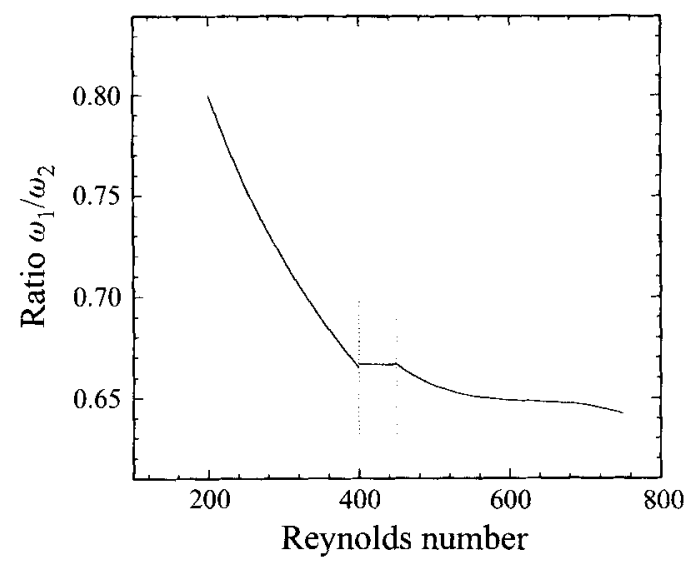

FigURE 5. Ratio of fundamental frequencies $\omega_{1} / \omega_{2}$ as a function of the Reynolds number.

of fundamental frequency $\omega_{1}$ sets in. At higher Reynolds numbers, another flow instability occurs leading to a second supercritical Hopf bifurcation. A second fundamental frequency $\omega_{2}$ appears, and the flow regime becomes quasi-periodic with two fundamental frequencies $\omega_{1}$ and $\omega_{2}$ and their linear combinations (Guzmán \& Amon 1994a).

We investigate the evolution of the fundamental frequency ratio, $\omega_{1} / \omega_{2}$, plotted in figure 5 as a function of the Reynolds number. The $\omega_{1} / \omega_{2}$ ratio is, in general, an irrational number, implying that both frequencies are incommensurate and the signal is aperiodic. This ratio decreases as the Reynolds number is increased until reaching asymptotically a plateau that coincides with the appearance of the frequency-locking phenomenon at $R e \approx 400$. Frequency-locking phenomena have also been reported in Rayleigh-Bénard convective flows (Gollub \& Benson 1980) and, recently, in twodimensional oscillatory flows around a circular cylinder (Vittori \& Blondeaux 1993). In these flows, further increases in the Reynolds number lead to chaotic flow regimes, whereas in the converging-diverging channel flow, a third supercritical Hopf bifurcation occurs for a Reynolds number of about 500 (Guzmán \& Amon 1994a). This leads to a quasi-periodic attractor with three fundamental frequencies $\omega_{1}, \omega_{2}$, and $\omega_{3}$ and their linear combinations.

\subsection{Pseudophase space representations}

The pseudophase space representation technique is based on time-delay reconstructions and allows the reconstitution of full-phase space behaviour from observable scalar time series (Packard et al. 1980; Takens 1981). For a scalar time series $z\left(t_{k}\right)$ of an observable $z(t)$, where $k \in K, K:=\left\{k \in N_{0} ; k<M_{d a t}\right\}$ and $M_{d a t}$ is the number of data points, state vectors in the reconstructed $n$-dimensional phase space are given by

$$
F\left(t_{s}\right)=\left(z\left(t_{s}\right), z\left(t_{s}+\tau\right), z\left(t_{s}+2 \tau\right), \ldots, z\left(t_{s}+(n-1) \tau\right)\right),
$$

where $s \in S, t_{s}=s \phi_{a}, S \in\left\{N_{0} ; s<M_{d a t}-(n-1) \tau / \phi_{a}\right\}, \phi_{a}$ is the sampling time of the continuous signal, $\tau$ is the time delay which is a multiple of $\phi_{a}$, and $n$ is the embedding dimension. If the attractor of the system in the original full space is $m$ dimensional, then the $n$-dimensional reconstructed attractor in the pseudophase space will have the same invariant properties, such as fractal dimensions and Lyapunov exponents, for all $n$ over a minimum value that is not greater than $2 m+1$ (Takens 1981). The choice of a proper time delay, $\tau$, and a sufficiently large embedding dimension, $n$, which are not 


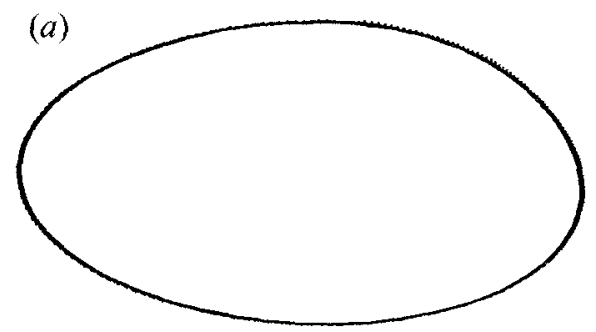

(c)

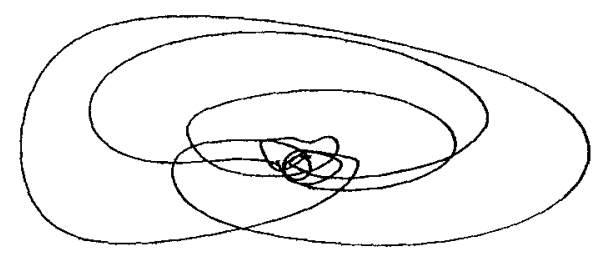

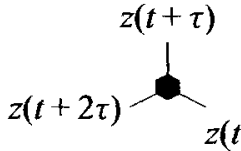

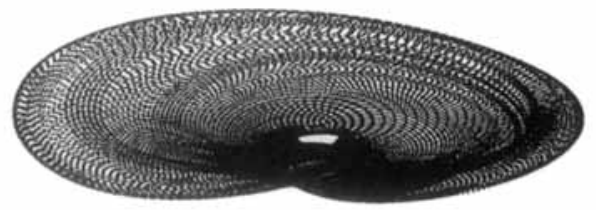

(d)

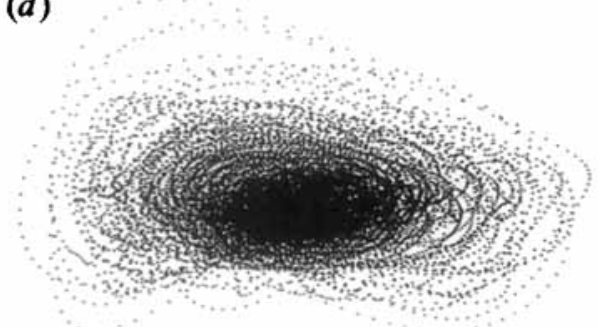

FIGURE 6. Three-dimensional pseudophase space representations based on time-delay reconstructions of the $U$-velocity at point $(x / h, y / h, z / h)=(4.67,3.33,0.0):(a) R e=150 ;(b) \operatorname{Re}=250 ;(c) \operatorname{Re}=400$; (d) $R e=850$.

known a priori, is not trivial for time series. Given a time series with an infinite number of discrete data points, without noise and without restrictions in resolution, the time delay can be chosen almost arbitrarily. However, in practice, we increase the reconstruction dimension until the invariant properties do not change.

The reconstructed attractors in the three-dimensional pseudophase space are shown in figure 6 for different flow regimes at $(x / h, y / h, z / h)=(4.67,3.33,0.0)$. In this figure, $z(t), z(t+\tau)$, and $z(t+2 \tau)$ represent the components of the state vector obtained from the streamwise $U$-velocity at a characteristic point in the converging-diverging channel centreline. The time delay $\tau$ for all the reconstructions presented here is twice the sampling non-dimensional time $\phi_{a}=0.023445$. The number of data points is $M_{d a t}=10001$ for all Reynolds numbers but $R e=850$ for which $M_{d a t}=35001$. Figures $6(a)$ and $6(b)$ show the fundamental limit cycle representing the periodic flow behaviour for $R e=150$ and the quasi-periodic flow obtained for $R e=250$, respectively. The flow at $R e=150$, represented by a single loop in the threedimensional pseudophase space, has evolved from a steady state to a limit cycle or periodic attractor through a first Hopf bifurcation. The orbits of the quasi-periodic flow with incommensurate fundamental frequencies at $R e=250$ lie on the surface of a $T^{2}$ torus. The deformed shape of this torus is due to the existence of many harmonics of the two fundamental frequencies (Guzmán \& Amon 1994a). Figure 6(c) shows the frequency-locking flow phenomenon that occurs for $R e=400$ as suggested by the plateau depicted in figure 5. The orbits of this flow, located on the surface of a $T^{2}$ torus, form a closed loop which verifies the locking of the two fundamental frequencies in the ratio $\omega_{1} / \omega_{2}=2 / 3$.

As the Reynolds number is increased further (e.g. $R e=500$ ), the quasi-periodic $T^{2}$ torus evolves to a $T^{3}$ torus. Figure $6(d)$ shows the reconstructed attractor for $R e=850$ 


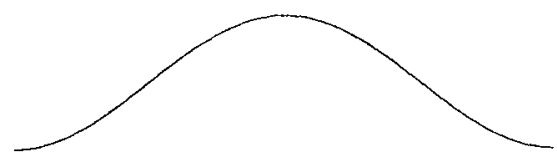

........
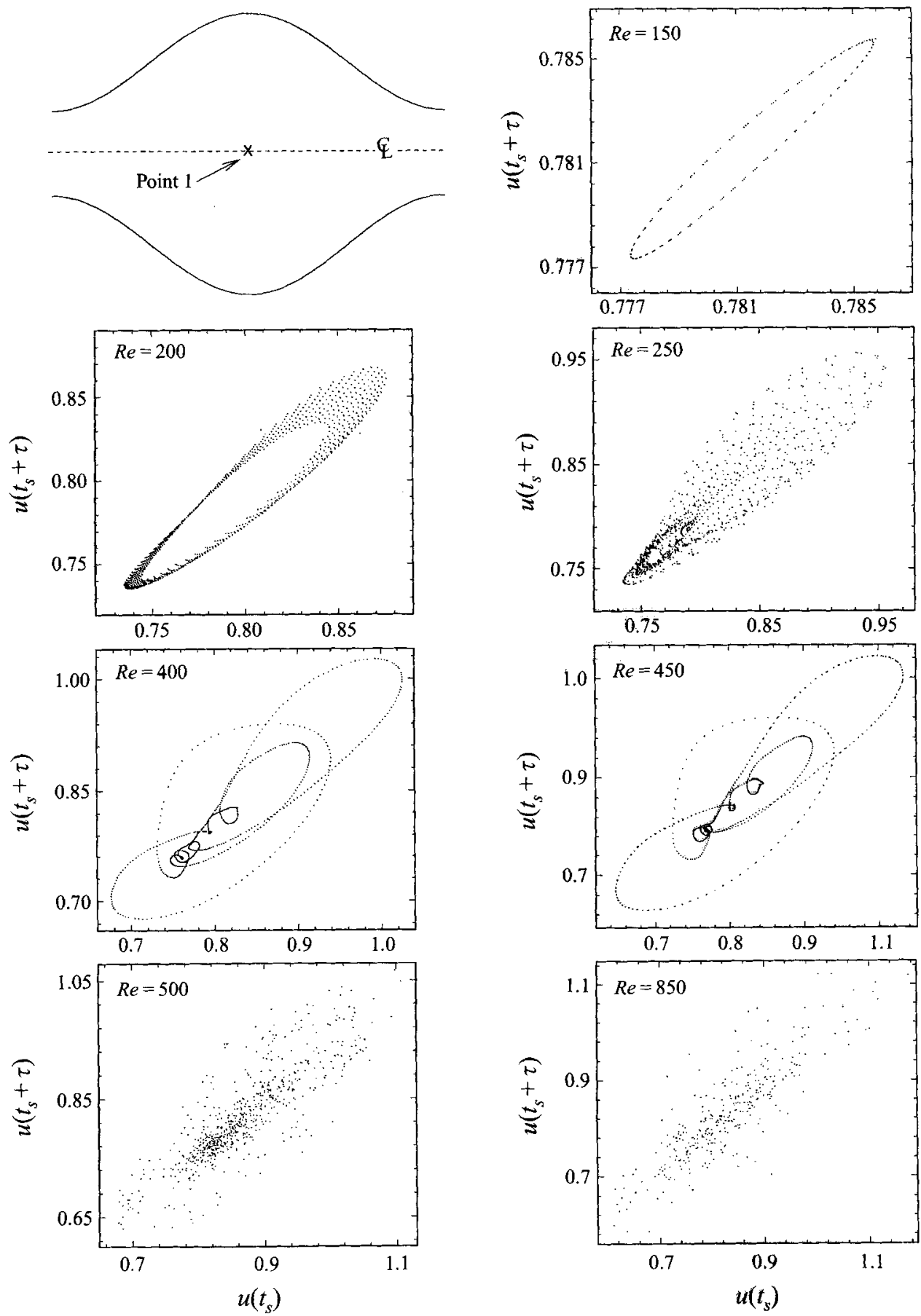

FIGURE 7. Poincare sections for the Reynolds number range from 150 to 850 at point 1 : $(x / h, y / h, z / h)=(4.67,3.33,0.0)$. 
where the preceding $T^{3}$ torus at $R e=500$ has been broken up into a strange attractor. Even though these orbits seem to follow random trajectories, they represent a deterministic chaotic behaviour.

\subsection{Poincaré sections}

The Poincaré section technique enables a systematic reduction in the representation of the system complexity by means of converting a continuous-time evolution into a discrete-time mapping and, consequently, reducing the problem dimensionality. Thus, trajectories with complicated appearance in a three-dimensional phase space could be depicted as trajectories inscribed on a $T^{2}$ torus. Although the attractor obtained in the new phase space is not identical to the original one, the new attractor's representation by a Poincaré section does retain the same topological properties as the flow from which it originates (Bergé et al. 1986).

To construct the Poincaré sections, we follow the method proposed by Bergé et al. (1986) of stroboscoping a time signal to determine the points in the Poincare section. This approach enables us to use the evolution trajectories on the phase space obtained from direct numerical simulations of the Navier-Stokes equations instead of data from evolution equations. We first construct a state vector $F\left(t_{s}\right)=\left\{u\left(t_{s}\right), u\left(t_{s}+\tau\right)\right\}$ based on the time delay reconstruction, where $t_{s}$ and $\tau$ are associated to the sampling and delay times, respectively, as outlined in $\$ 3.2$. Then, we obtain a trajectory of the continuoustime evolution within the pseudophase space, defined by the state vector and the sampling time such that each point of the trajectory is given by $\left\{u\left(t_{s}\right), u\left(t_{s}+\tau\right), t_{s}\right\}$. The Poincaré section is determined by the successive intersection points between this trajectory in the pseudophase space and a plane perpendicular to the time axis at intervals of time $T_{1}$, where $T_{1}=2 \pi / \omega_{1}$ is the period associated with the first fundamental frequency $\omega_{1}$ of either the periodic or quasi-periodic flow and it is the time interval necessary to complete one cycle on the attractor. We perform long enough computational simulations to obtain the many points required for accurate Poincaré map representations.

Figures 7 and 8 depict the Poincare sections corresponding to continuous-time evolutions at two typical points in the computational domain for the Reynolds number range from 150 to 850 . Poincaré sections with well-organized structures are obtained for Reynolds number flows up to 450 . For the time periodic flow at $R e=150$ and the frequency-locking flow at $R e=400$, the Poincare sections are composed by a discrete set of intersection points tracing a closed orbit. A blow up of particular regions shows more clearly that seemingly continuous sets of points are in fact discretely distributed on the closed orbit within the Poincaré section. The time signal for $R e=400$ contains two commensurate fundamental frequencies $\omega_{1}$ and $\omega_{2}$ whose ratio $\omega_{1} / \omega_{2}$ is a rational number (Guzmán \& Amon 1994a). Therefore, the Poincaré section is composed of a finite number of points which correspond to interceptions at same locations in the Poincaré section.

For quasi-periodic flow regimes, e.g. $R e=200$ and $R e=250$, the ratio of the fundamental frequencies $\omega_{1} / \omega_{2}$ is an irrational number and, therefore, the trajectory of points within the pseudophase space intercepts the Poincaré section at different locations as depicted in figures 7 and 8 . Because of the many subharmonics, superharmonics and linear combinations of the two fundamental frequencies of these quasi-periodic flows (Guzmán \& Amon 1994a), trajectories within the pseudophase space densely cover the surface of the deformed $T^{2}$ torus shown in figure $6(b)$. Thus, the successive intersections of these trajectories form a Poincare section with a dense cloud of points that partially fill the bounded cross-sectional area of the deformed 

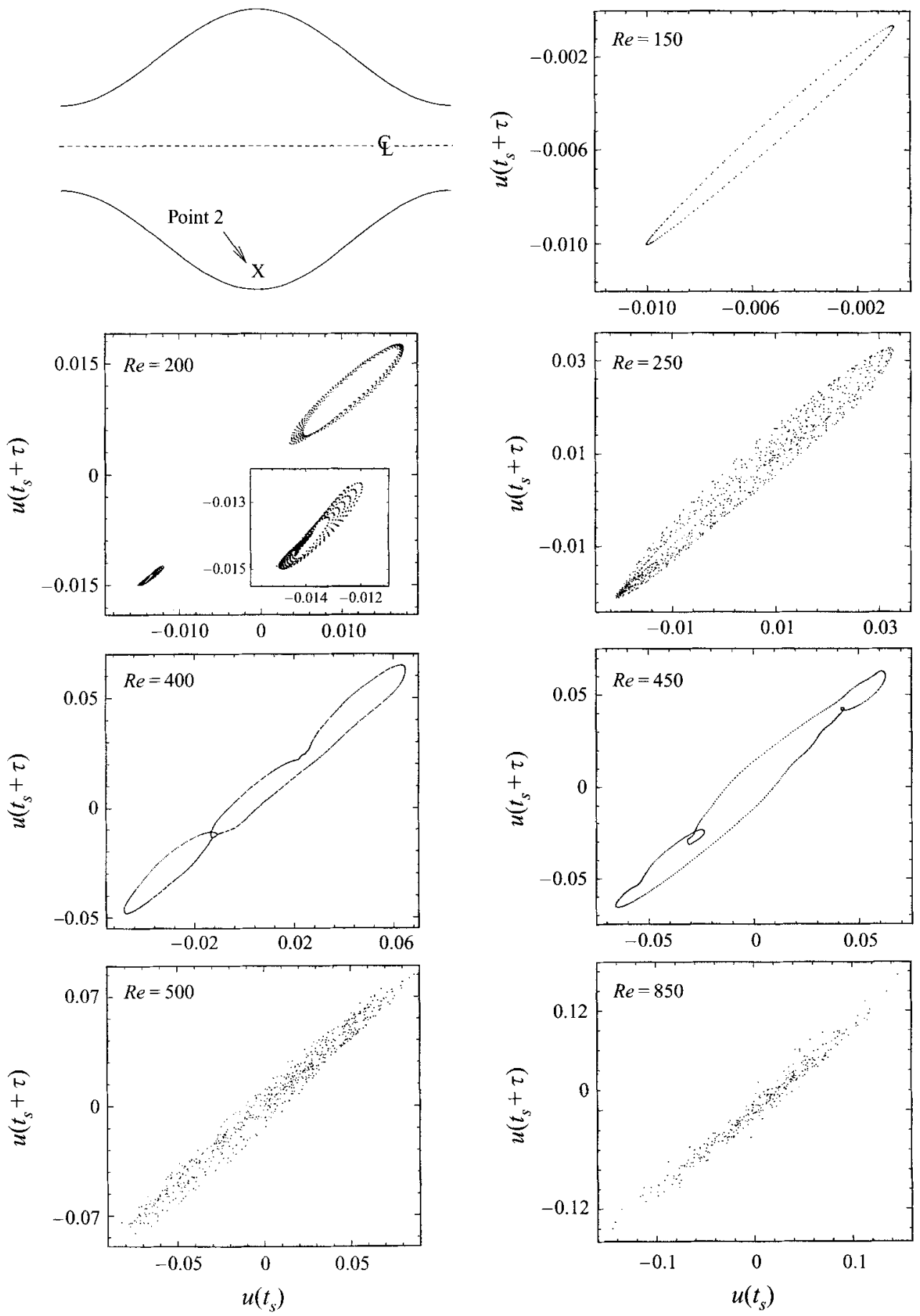

Figure 8. Poincaré sections for the Reynolds number range from 150 to 850 at point 2: $(x / h, y / h, z / h)=(4.67,0.18,0.0)$. 
(a)
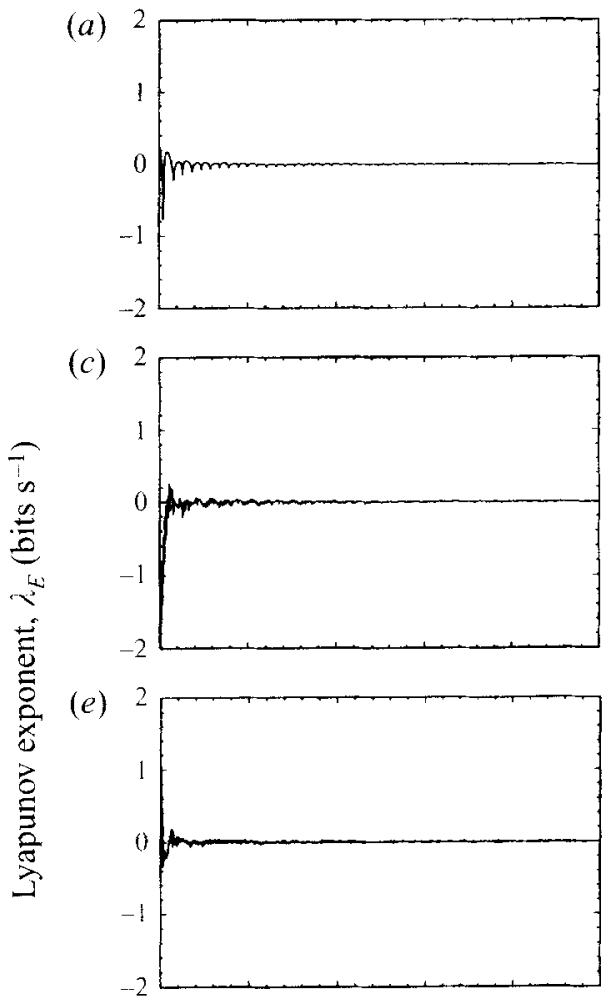

$(g)$

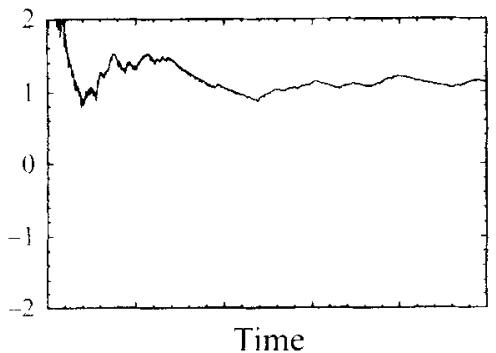

(b)

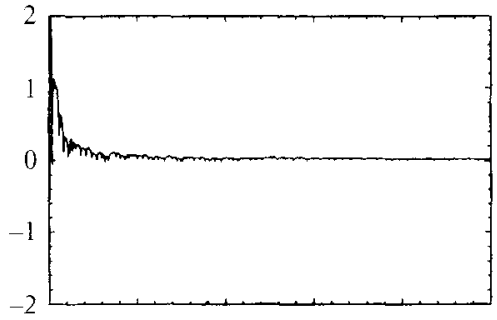

(d)

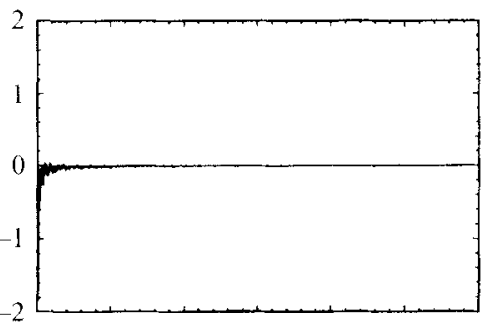$$
-2
$$

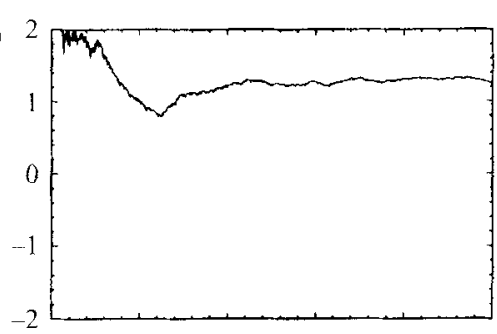

(h)

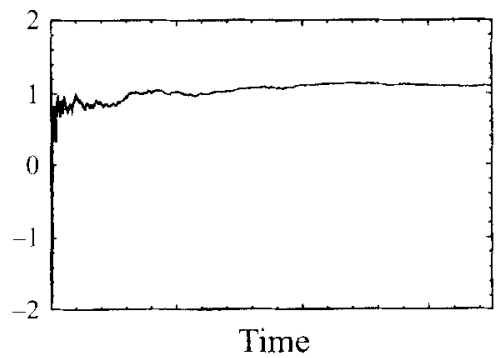

FIGURE 9. Temporal convergence of the largest Eulerian Lyapunov exponent $\lambda_{E}$ : $(a) R e=150$; (b) $\operatorname{Re}=250 ;(c) \operatorname{Re}=400 ;$ (d) $\operatorname{Re}=450 ;$ (e) $\operatorname{Re}=500 ;(f) \operatorname{Re}=600 ;$ (g) $\operatorname{Re}=750 ;$ (h) $R e=850$.

torus, becoming more crowded as longer time trajectories are considered. In the limit of very large simulation times, an infinite number of intersection points in the Poincaré section will fill this bounded region. For higher-Reynolds-number flows, Poincaré sections are composed by a non-structured and diffuse sequence of interception points such as for $R e=500$ which is a flow regime with a $T^{3}$ torus attractor and for $R e=850$ which corresponds to an aperiodic flow regime represented by a broadband Fourier power spectrum (Guzmán \& Amon 1994a).

A quantitative measure of the onset of chaos and the long-term persistent unpredictable nature of chaotic flow regimes is presented next from estimates of Eulerian Lyapunov exponents and fractal dimensions. 


\subsection{Eulerian Lyapunov exponents}

The Eulerian Lyapunov exponents spectrum, $\left\{\lambda_{E}\right\}$, is the most complete possible characterization of the geometric properties of an attractor and its dynamical flow properties for dynamical features such as fractal dimension and entropy can be calculated from $\left\{\lambda_{E}\right\}$. The Eulerian Lyapunov exponent measures the long-time average exponential growth or decay of infinitesimal perturbations to a phase space trajectory, i.e. it measures the sensitivity of the system to initial conditions in the phase space. Different algorithms have been proposed and developed to calculate Eulerian Lyapunov exponents (Wolf et al. 1985; Eckmann et al. 1986; Briggs 1990; Vastano \& Moser 1991; Keefe, Moin \& Kim 1992; Barna \& Tsuda 1993; Rosenstein, Collins \& De Luca 1993). An attractor in an $N$-dimensional phase space has $N$ Eulerian Lyapunov exponents. Researchers have demonstrated that, in certain flow regimes, the Navier-Stokes equations can be represented by a low-dimensional attractor whose dimension increases with the Reynolds number (Brandstater \& Swinney 1987; Vastano \& Moser 1991; Pulliam \& Vastano 1993). While the attractor may be low dimensional in the chaotic regime near the threshold of turbulence, in regimes of developed turbulence, its dimension soon becomes very large (Gaspard \& Wang 1993). If an attractor has one or more positive Eulerian Lyapunov exponents, $\lambda_{E}$, perturbations on the attractor can grow exponentially fast in the directions of the positive exponents. In such a case, the attractor is said to be chaotic and is called a strange attractor (Bergé et al. 1986). A negative $\lambda_{E}$ implies an exponential decay towards the attractor. Given a non-positive Eulerian Lyapunov exponent spectrum, ordered as $\lambda_{E}=\lambda_{1} \geqslant \lambda_{2} \geqslant$ $\lambda_{3} \geqslant \ldots \geqslant \lambda_{N}$, when all exponents are negative, it implies a fixed point attractor; when $\lambda_{E}=0$ and all other exponents are negative, it corresponds to a periodic attractor, and $\lambda_{E}=\lambda_{2}=0$ represents a quasi-periodic attractor.

To calculate the largest Eulerian Lyapunov exponent, $\lambda_{E}$, we implemented the algorithm proposed by Wolf et al. (1985) for time series and we applied to Eulerian velocity evolutions obtained from direct numerical simulations of convergingdiverging channel flows. We performed extensive trials with different embedding dimensions to find the smallest reliable embedding dimension to calculate the Eulerian Lyapunov exponents. The embedding dimension for all the Reynolds numbers reported is $n=4$. All trials with higher embedding dimensions lead to the same qualitative behaviour, and quantitative differences are no greater than $4 \%$. Figure 9 shows the temporal convergence of $\lambda_{E}$ for the Reynolds numbers range of 150-850. A zero value of the Eulerian Lyapunov exponent is obtained for non-chaotic selfsustained oscillatory flows up to Reynolds numbers of 500 (figures $9 a-c$ ). These zero Eulerian Lyapunov exponents indicate the exponential decay towards the attractor of trajectories on the phase space when small changes on the initial conditions occur. Positive Eulerian Lyapunov exponents, obtained for Reynolds numbers of 600, 750, and 850 and depicted in figures $9(f)-9(h)$, verify chaotic flow behaviours.

Table 1 shows the largest Eulerian Lyapunov exponent, $\lambda_{E}$, as a function of the Reynolds number. The estimates of $\lambda_{E}$ are given in terms of the asymptotically converged mean value and the standard deviation of the fluctuating values of $\lambda_{E}(t)$. The large positive Eulerian Lyapunov exponents, attained consistently for Reynolds numbers greater or equal than 550, indicate that the flow remains chaotic in this converging-diverging channel and verify the qualitative results provided by the autocorrelation functions and the pseudophase space representations. Numerical simulations of unforced two-dimensional compressible flows past airfoils predict increases of $\lambda_{E}$ as a function of the Reynolds number after the onset of chaos (Pulliam 


$\begin{array}{ccc}R e & \lambda_{E} & \mathrm{SD} \\ 150 & 0.0001927 & 0.004935 \\ 250 & 0.0000906 & 0.000076 \\ 400 & 0.0023254 & 0.007319 \\ 450 & -0.0021815 & 0.002453 \\ 500 & 0.0010185 & 0.003175 \\ 550 & 0.2367243 & 0.007316 \\ 600 & 1.274962 & 0.034339 \\ 750 & 1.108400 & 0.047150 \\ 850 & 1.102538 & 0.020658\end{array}$

TABLE 1. Asymptotically-converged mean value of the largest Lyapunov exponent, $\lambda_{E}$, and standard deviation, $\mathrm{SD}$, of the fluctuating values of $\lambda_{E}(t)$ for different Reynolds numbers

\& Vastano 1993) whereas numerical calculations performed for a logistic map give a similar, but continual, distribution of the Lyapunov exponents as a function of a control parameter (Ruelle 1992).

\subsection{Fractal dimensions}

Simple attractors, such as fixed points for steady flows, periodic orbits for onefrequency oscillatory flows and $N$-tori for $N$-frequency motions, attract asymptotically all trajectories sufficiently close. The primary characteristic of chaos is that the flow is unstable on the attractor. Consequently, infinitesimal perturbations grow at an exponential rate in one or more directions of the attractor. A dynamical system with $N$ degrees of freedom has a strong attractor when it exhibits a chaotic evolution on the $N$-dimensional phase space. Therefore, a strange attractor is chaotic when there is at least one direction of exponential growth (Grassberger \& Procaccia 1983; Brandstater \& Swinney 1987; Vastano \& Moser 1991).

A useful parameter to characterize the geometry of a strange attractor is its fractal dimension. The fractal dimension measures the degrees of freedom that are relevant to the flow dynamics and that are necessary to capture the main flow features. The Hausdorff-Besikovich fractal dimension, $D$, is a purely geometric measure of the fractal local structure of the attractor. It is independent of the frequency at which a trajectory visits the different parts of the attractor. However, $D$ is difficult to calculate and impractical for high-dimensional systems. Instead, the information dimension, $d_{I}$, and the correlation dimension, $v$, are more useful measures of the local structure of a strange attractor. These fractal dimensions are related by the inequalities $v \leqslant d_{I} \leqslant D$, and, in most cases, the correlation dimension $v$ can be measured more easily than either $d_{I}$ or $D$. The fractal dimension can also be evaluated from the spectrum of Eulerian Lyapunov exponents. Larger positive Eulerian Lyapunov exponents imply that more directions of instabilities exist on the attractor, causing a higher fractal dimension. The relationship between $D$ and $\lambda_{E}$ is given by the Kaplan-Yorke conjecture (Frederickson et al. 1983) which relates the Lyapunov exponent spectrum $\left\{\lambda_{E}\right\}$ to the dimension of the attractor, $D_{\lambda}$, by

$$
D_{\lambda}=m+\left(\sum_{i=1}^{m} \lambda_{i} /\left|\lambda_{m+1}\right|\right),
$$

where $m$ is the largest number for which $\sum_{i=1}^{m} \lambda_{i}$ is positive.

Experimental investigations and numerical simulations, in closed and open flow systems, have shown evidence that chaotic, strange attractors are low dimensional at 
the onset of chaos or in flow regimes of weak turbulence. The dimension of the attractor increases gradually as the system moves towards a developed turbulence (Brandstater \& Swinney 1987; Vastano \& Moser 1991; Pfister et al. 1992; Pulliam \& Vastano 1993; Broze \& Hussain 1994). For example, the Taylor-Couette flow attractor is low dimensional $(n=2)$ at the onset of turbulence, and its dimension increases up to a value higher than 4 in turbulent flow regimes (Brandstater \& Swinney 1987). Vastano \& Moser (1991) corroborated the previous experimental results in Taylor-Couette flows and found that the fractal dimension increases continuously as the Reynolds number is increased in the turbulent regime. Pulliam \& Vastano (1993) found that the transition to chaos in open unforced two-dimensional flows past an airfoil is gradual and low dimensional. This transition occurs as if it were governed by a one-dimensional map since the bifurcation scenario is similar to that of the logistic map with a period-doubling cascade and windows of periodicity embedded by chaos. However, the fractal dimension $D_{\lambda}$ increases monotonically as the Reynolds number is increased beyond the onset of chaos.

We examine temporal flow evolutions with seemingly erratic behaviour on both short- and long-term timescales in terms of the Hurst's rescaled range analysis. The Hurst's rescaled range analysis $(R / S)$ is a statistical method for analysing a time signal and for estimating its fractal dimension, $d$. The records are characterized by the Hurst's exponent, $H$, and the trace of the record with a fractal dimension $d=2-H$ (Feder 1988). The significance of $d$ is that it describes the relation between the variance of the signal and the timescale. No assumptions are made on the underlying physics of the system from which the signal is observed. It is, therefore, a purely statistical tool. Fractal systems have neighbour-to-neighbour correlations at all scale levels. When neighbouring elements in a time series are positively correlated, that is $1<D<1.5$, the measured variance drops less rapidly as the resolution is decreased. If a signal $\xi(t)$ is divided into intervals of time, $\Delta t$, and the mean as well as the variance is calculated for each interval, then, there will be a lower variance when the interval length is increased. Therefore, the relationship between the variance and the sample time may fit a power law, and if so, the fractal dimension of the time series can be calculated by the Hurst's rescaled range analysis (Schepers, van Beck \& Bassingthwaighte 1992).

For converging-diverging channel flows, we calculate the fractal dimension, $d$, of the $U$-velocity time series using Hurst's empirical law and rescaled range analyses proposed by Hurst (1951) and described by Feder (1988). The time series contains 70001 data points for $R e=150$ and 140001 data points for $R e=850$. Figure 10 shows the log- $\log$ plot of $R(\theta) / S(\theta)$ vs. $\theta$, where $R(\theta) / S(\theta)$ is the rescaled range;

$$
R(\theta)=\max \{X(t, \theta)\}-\min \{X(t, \theta)\}, 1 \leqslant t \leqslant \theta,
$$

is the range defined as the difference between the maximum and the minimum accumulated influx $X(t, \theta)=\Sigma_{\alpha=1}^{t}\left\{\xi(\alpha)-\langle\xi\rangle_{\theta}\right\}$ from the mean flow $\langle\xi\rangle_{\theta}=(1 / \theta) \Sigma_{t=1}^{\theta} \xi(t)$ for a given interval of time $\theta$; and, $S(\theta)$ is that standard deviation. The rescaled range, $R(\theta) / S(\theta)$, for each time interval $\theta$ is plotted and the slope of the best fitted curve represents the Hurst's exponent, $H$. The plot of $R(\theta) / S(\theta) v s . \theta$ for $R e=150$, shown in figure $10(a)$, depicts two distinct regions. The Hurst's exponent in the first region is approximately equal to one as given by the slope of the least-squares fitted curve. According to $d=2-H$, the fractal dimension, $d$, is also close to one. This represents a high correlation or a long-term persistence in the velocity evolution and confirms the nature of the periodic attractor obtained for $R e=150$. The second region of this graph $\left(\theta>\theta^{*}=20\right)$ is not meaningful to calculate the Hurst's exponent $H$ for it corresponds to frequencies smaller than the fundamental frequency of this periodic flow regime. 


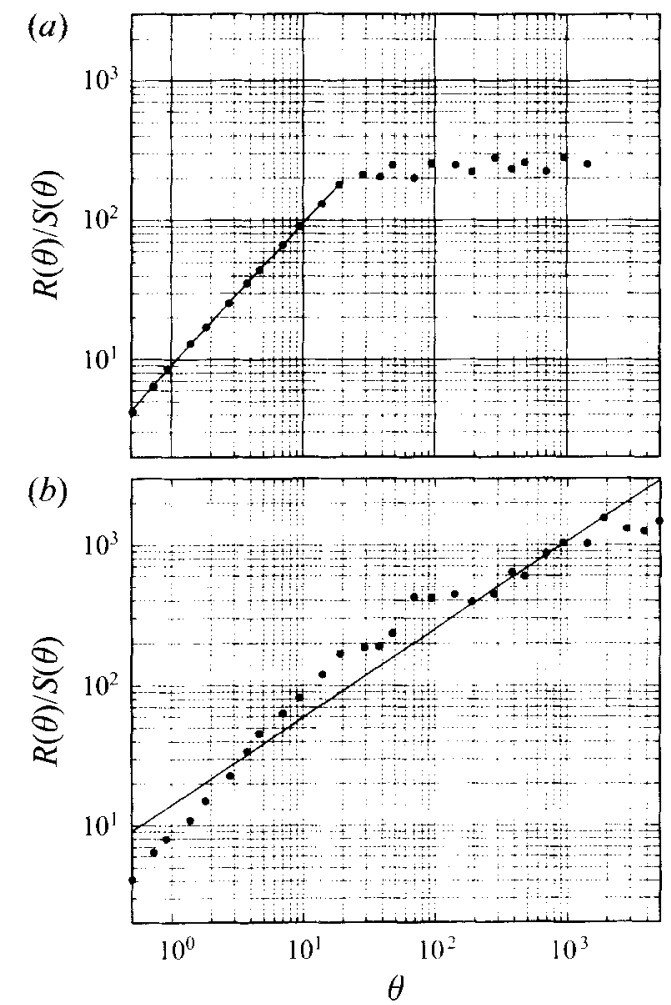

Figure 10. $\log -\log$ plot of the rescaled range $R(\theta) / S(\theta)$ es. $\theta$. The slope determines the Hurst's exponent $H$ for the fractal dimension $d=2-H:(a) R e=150 ;(b) R e=850$.

Figure $10(b)$ presents a $\log -\log$ plot of $R(\theta) / S(\theta)$ vs. $\theta$ for $R e=850$. The slope of the least-squares fitted curve for the whole region gives a Hurst's exponent of $H=0.623928$ which implies that this flow velocity evolution has a lower correlation as well as less memory over time than the flow at $R e=150$. It also indicates the long-term persistent, unpredictable, characteristic of this chaotic flow regime, which assures an unpredictable flow in the future based on the unpredictability of the flow in the present. The fractal dimension increases from $d=1$ as the Reynolds number is increased in the chaotic flow regime and, for this Reynolds number of 850 , the fractal dimensions is $d=1.376072$.

\section{Lagrangian trajectories on laminar and transitional flow regimes}

In $\S 3$ we have shown the existence of chaotic flows within strange attractors which are originated from Eulerian velocity fields $\boldsymbol{u}\left(\boldsymbol{x}_{p}, t\right)$, where $p$ are fixed positions on the converging-diverging channel. When the flow is described in a Lagrangian frame, we determined the fluid particle trajectories by integrating the differential equation $\mathrm{d} \boldsymbol{x} / \mathrm{d} t=\boldsymbol{u}(\boldsymbol{x}, t)$, where $\boldsymbol{u}(\boldsymbol{x}, t)$ denotes the Eulerian velocity field obtained from direct numerical simulation (DNS) of the time-dependent Navier-Stokes equation solver. In this case, a sensitive dependence on initial conditions indicates the presence of Lagrangian chaos, which implies that nearby trajectories diverge exponentially fast (Danielson \& Ottino 1990). Researchers have investigated the relation between the onset of Lagrangian chaos and Eulerian chaos, the value of their Lyapunov exponents, 

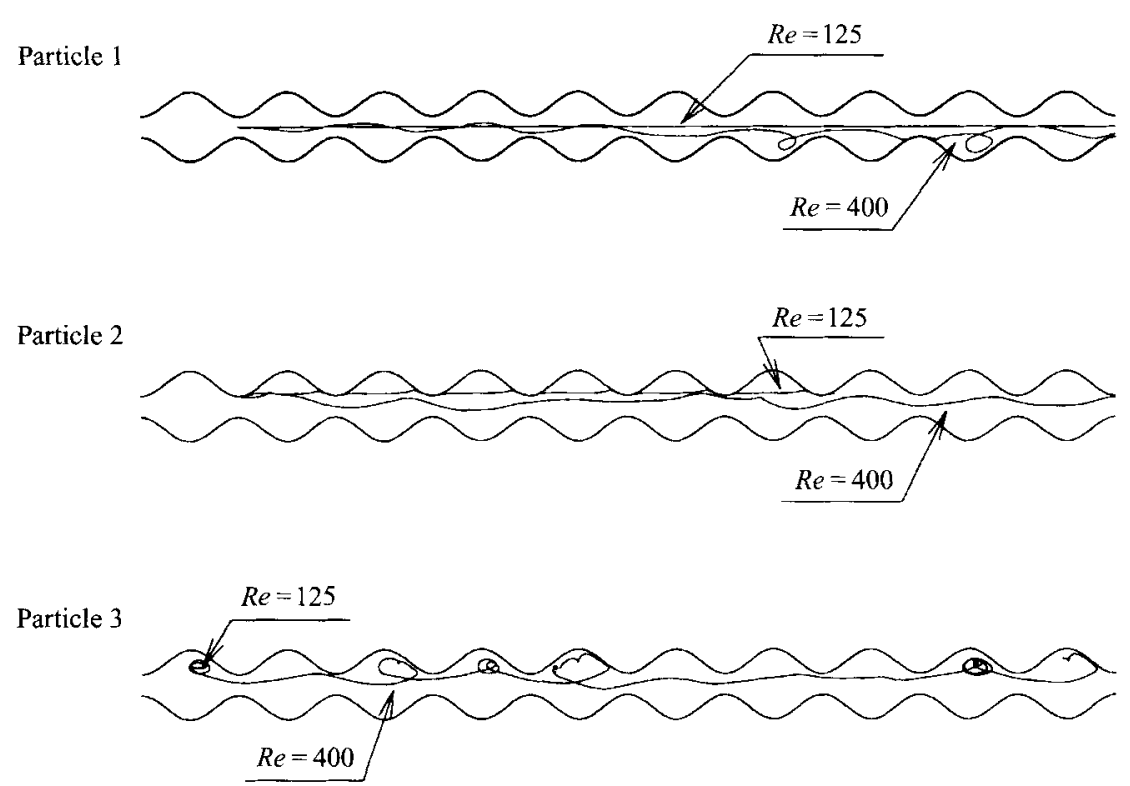

FIGURE 11. Lagrangian trajectories of three fluid particles for Reynolds numbers of 125 and 400 .

and the effect that one form of chaos has on the other one (Babbiano et al. 1994; Danielson \& Ottino 1990; Guzmán 1995; Amon, Guzmán \& Morel 1996). One would expect that in the presence of a chaotic Eulerian velocity field, the particle motion would necessarily be chaotic. However, numerical studies have shown that there are exceptions to this seemingly general behaviour (Lorenz 1963; Crisanti et al. 1991).

Our results presented in previous sections demonstrate that the converging-diverging channel flow evolves to a regime of Eulerian chaos from a well-organized velocity field. The positive Eulerian Lyapunov exponents obtained for Reynolds number flows equal to or higher than 550 indicate chaotic flow regimes which are also visualized by the pseudophase space and the Poincaré section representations. Our next objective is to characterize the laminar and transitional flow regimes from a Lagrangian view point, in terms of Lagrangian trajectories for single fluid particles throughout the flow domain and for pairs of fluid particles located in the vortical regions.

Lagrangian trajectories of three test particles (denoted as particle 1,2 and 3) starting at different regions of the computational domain and passing through many channel furrows for Reynolds numbers of 125 and 400 are shown in figure 11. For the steady flow regime at $R e=125$, each test particle follows the same path in each furrow of the channel and test particle trajectories coincide with the streamlines of the Eulerian velocity field. For the time-dependent flow regime at $R e=400$, test particles follow more complex trajectories, as shown in figure 11 . They are not confined to move in small regions and can fill the whole computational domain. For example, particle 1 starts its motion in the middle of the channel, continues in the vortex region and, finally, returns to the main flow. Similarly, particle 3 starts its motion in a vortical region, moves to downstream furrows and convects through different vortices in a more complex trajectory.

Figure 12 depicts Lagrangian trajectories of five pairs of test particles located initially in the vortex region for Reynolds numbers of 125 and 150 . The initial and final positions of the test particles are denoted by the characters $i$ and $f$, respectively. The trajectory of each test particle in the pair is composed by a sequence of points 
(a)
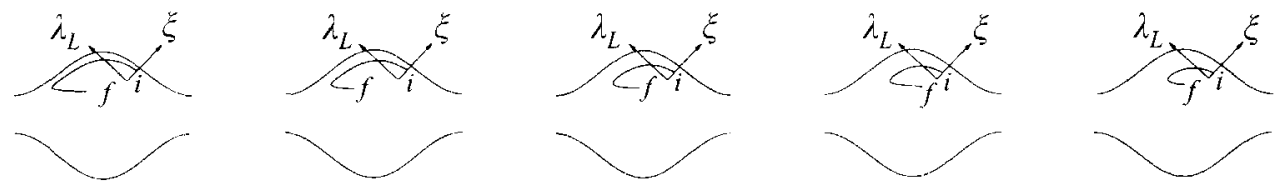

(b)

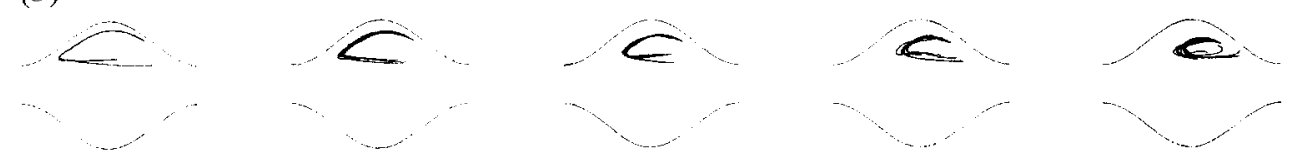

FIGURE 12. Lagrangian trajectories of five pairs of test fluid particles: $(a) \operatorname{Re}=125 ;(b) \operatorname{Re}=150$.

representing its instantaneous position. The trajectories of two test particles in the pair are so close $\left(d / h \approx 10^{-5}-10^{-4}\right)$ that are indistinguishable in the figure. For $R e=125$, the test particles follow trajectories that coincide with the streamlines of the Eulerian steady flow regime (figure 12a). Lagrangian trajectories of test particles starting their motion in the vortex region remain within this region forever. A more complex pattern of Lagrangian trajectories is observed in figure $12(b)$ for $R e=150$, where pairs of particles sometimes remain in the vortex region but without repeating the same path and eventually convect downstream in the channel. These trajectories are synchronized with the oscillating motion of the vortices owing to the periodic self-sustained flow regime.

The chaotic nature of particle trajectories cannot be determined by just looking at the evolution patterns of particle pairs, even though complex paths could provide a qualitative indication of Lagrangian chaos (Ottino 1989). Chaotic trajectories can be quantified by the time-average divergence of trajectories of particles initially close which is given by the finite-time Lagrangian Lyapunov exponent (e.g. Rom-Kedar, Leonard \& Wiggins 1990). The spatial distribution of Lagrangian Lyapunov exponents allows us to determine regions of chaotic particle trajectories and to measure the strength of Lagrangian chaos (Amon et al. 1996). We found that the onset of Lagrangian chaos at $125<R e<150$ coincides with the first Hopf bifurcation and complex particle trajectories induce chaotic advection and mixing enhancement, which is verified by positive finite-time Lagrangian Lyapunov exponents and stretching rate distributions. We also demonstrated the existence of Lagrangian chaos in non-chaotic Eulerian converging-diverging flows and the onset of Eulerian chaos coinciding with the spreading of a strong Lagrangian chaotic regime (Amon et al. 1996).

\section{Three-dimensional flow simulations}

Our previous investigations have determined that the transition route to chaos in converging-diverging channel flows, through the Ruelle-Takens-Newhouse (RTN) scenario, obeys two-dimensional flow bifurcations (Guzmán \& Amon 1994a). This scenario was assumed to occur without being affected by three-dimensional spanwise flow disturbances that could potentially grow as a consequence of secondary instabilities. Experimental results reported by Nishimura et al. (1990), for the same converging-diverging channel geometry, show no evidence of flow three-dimensionality in the range of Reynolds number explored, which corroborates our previous assumption. However, stable three-dimensional states have been found in internal 
(a)
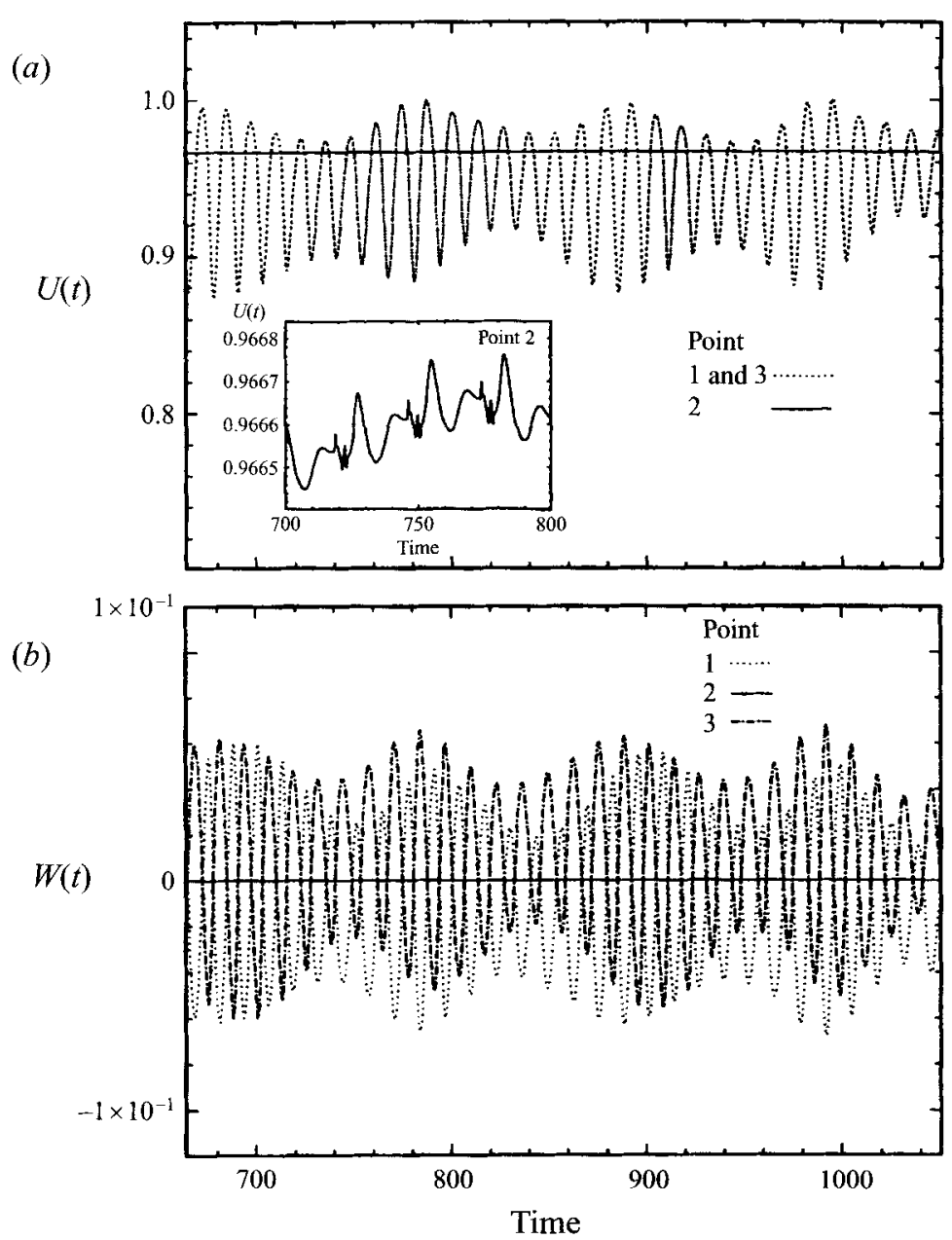

FIGURE 13. Time evolution of: (a) $U$-velocity; (b) $W$-velocity components at three characteristics points $P_{1}, P_{2}$ and $P_{3}$, for $R e=226$.

channel flows where geometric inhomogeneities or flow obstacles induce flow separations and inflectional velocity profiles (Amon \& Patera 1989; Batcho \& Karniadakis 1991; Schatz \& Swinney 1992). In these flows, the primary instability leads to two-dimensional flow oscillations that become self-sustained, and these selfsustained oscillatory flows are susceptible to three-dimensional disturbances at larger Reynolds numbers.

The converging-diverging channel also induces inflectional velocity profiles which are capable of triggering inviscid instabilities (Rayleigh 1945). Thus, our next objective is to determine the effect of the spanwise flow direction on the transition to chaos. We perform three-dimensional numerical simulations at moderate Reynolds number flow regimes for which DNS can resolve all temporal and spatial flow scales. Since DNS time-evolution calculations of three-dimensional transitional flows in complex geometries require massive computer memory and CPU times, we were able to simulate only a few typical flow regimes to date; nevertheless, these simulations enable us to investigate the effects of three-dimensionality in a limited range of Reynolds numbers. We investigate transitional flow regimes near the first and second Hopf bifurcations and present velocity histories, Fourier power spectra, velocity profiles, and 
(a)
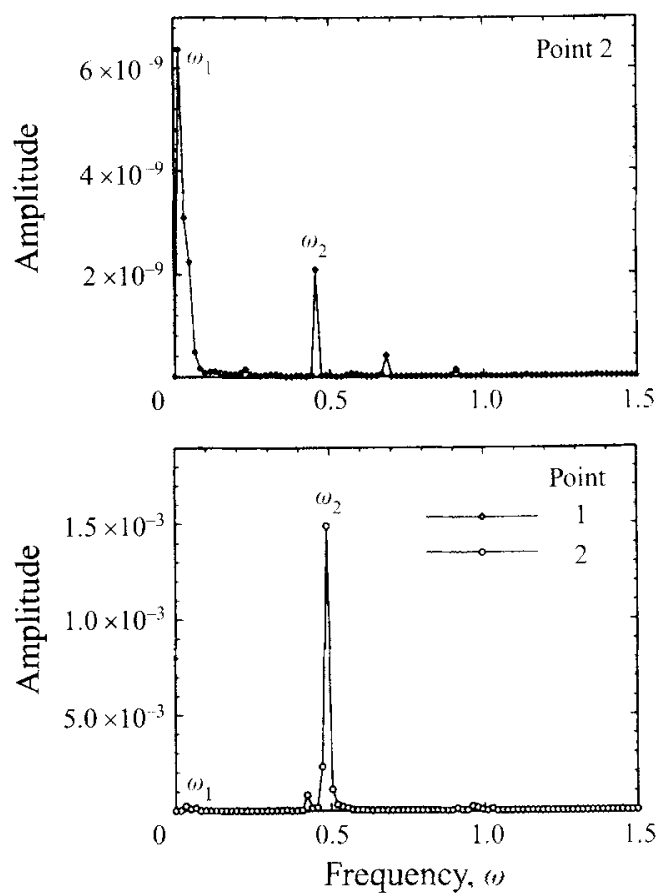

(b)
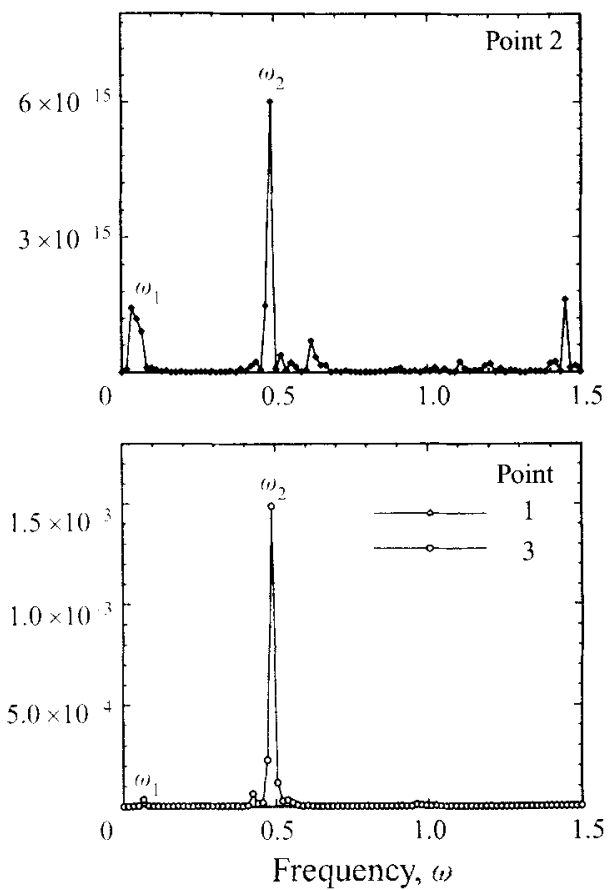

Figure 14. Fourier power spectra of the velocity components at $P_{1}, P_{2}$ and $P_{3}$ for $R e=226$ : (a) $U$-velocity; (b) $W$-velocity.

streamtracers in $\$ 5.1$, and we discuss the effects of three-dimensionality and spanwise boundary conditions in $\$ 5.2$.

\subsection{Three-dimensional transitional flow characterization}

In this section, we describe three-dimensional flow simulations in converging-diverging channels with non-slip spanwise boundary conditions at the sidewalls, in terms of velocity time series, Fourier power spectra, velocity profiles, and streamtracers. The time evolution of the streamwise $U$-velocity and the spanwise $W$-velocity components are shown at three characteristic points of the computational domain. The spatial positions of these points are indicated in figure $1 ; P_{1}$ and $P_{3}$ are located close to the sidewalls of the channel at $z / h=-24$ and $z / h=24$, respectively, and $P_{2}$ is within the symmetry plane at $z / h=0$.

The streamwise $U$-velocity is oscillatory with amplitudes depending on the spatial location within the channel (figure $13 \mathrm{a}$ ). The amplitude of the velocity oscillations at $P_{2}$ is about three orders of magnitude smaller than the amplitude at $P_{1}$ and $P_{3}$. The $U$-velocity oscillations at $P_{1}$ and $P_{3}$ have exactly the same magnitude and direction, which corresponds to a synchronized motion with a zero phase angle due to the symmetry of the channel with respect to both the crosswise $y$-direction and the spanwise $z$-direction. However, even though the $W$-velocity oscillations at $P_{1}$ and $P_{3}$ have the same magnitude, they have opposite directions (figure $13 b$ ). This means that fluid particles located symmetrically with respect to $z / h=0$ oscillate with equal frequencies and move either to or from the sidewalls of the channel. Furthermore, the amplitude of these flow oscillations decreases towards the centre plane and becomes zero at $P_{2}$ as a consequence of the asymmetric fluid motion along the spanwise $z$-direction.

The Fourier power spectra of the velocity time evolutions, shown in figures 14(a) 


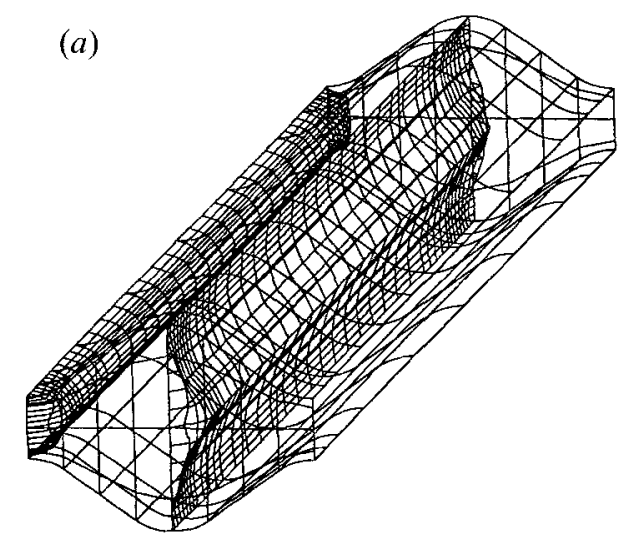

(b)

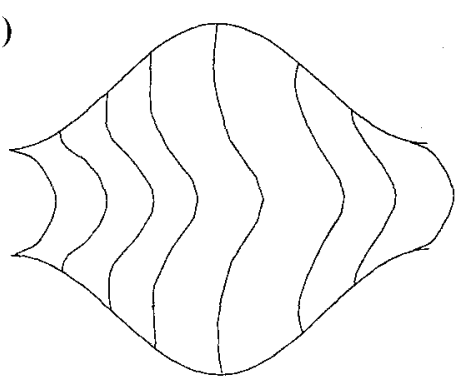

(c)
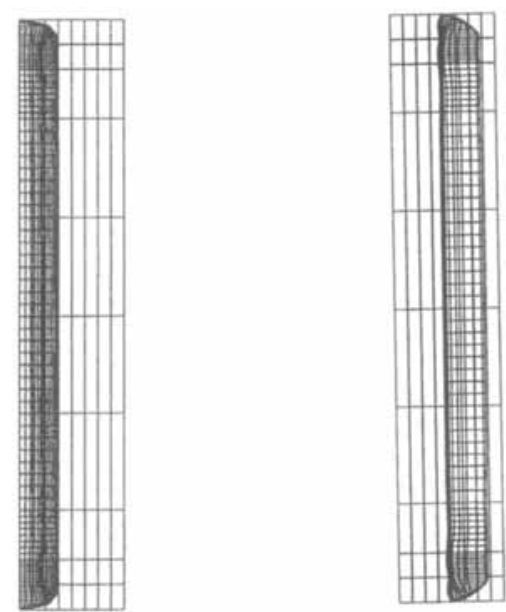

FIGURE 15. $U$-velocity profiles at $x / h=0$ and $x / h=4.67:(a)$ isometric view; $(b) z$-axis view; (c) $y$-axis view.

and $14(b)$, display two fundamental frequencies, $\omega_{1}$ and $\omega_{2}$, along with their linear combinations. These fundamental frequencies and the pattern of their linear combinations are the same as those obtained in the two-dimensional simulations corresponding to a quasi-periodic flow regime.

Figure $15(a)$ shows an isometric projection of $U$-velocity profiles at planes perpendicular to the streamwise $x$-direction of the channel. The $z$-axis view of these velocity profiles, shown in figure $15(b)$, depicts a nearly parabolic velocity profile at 

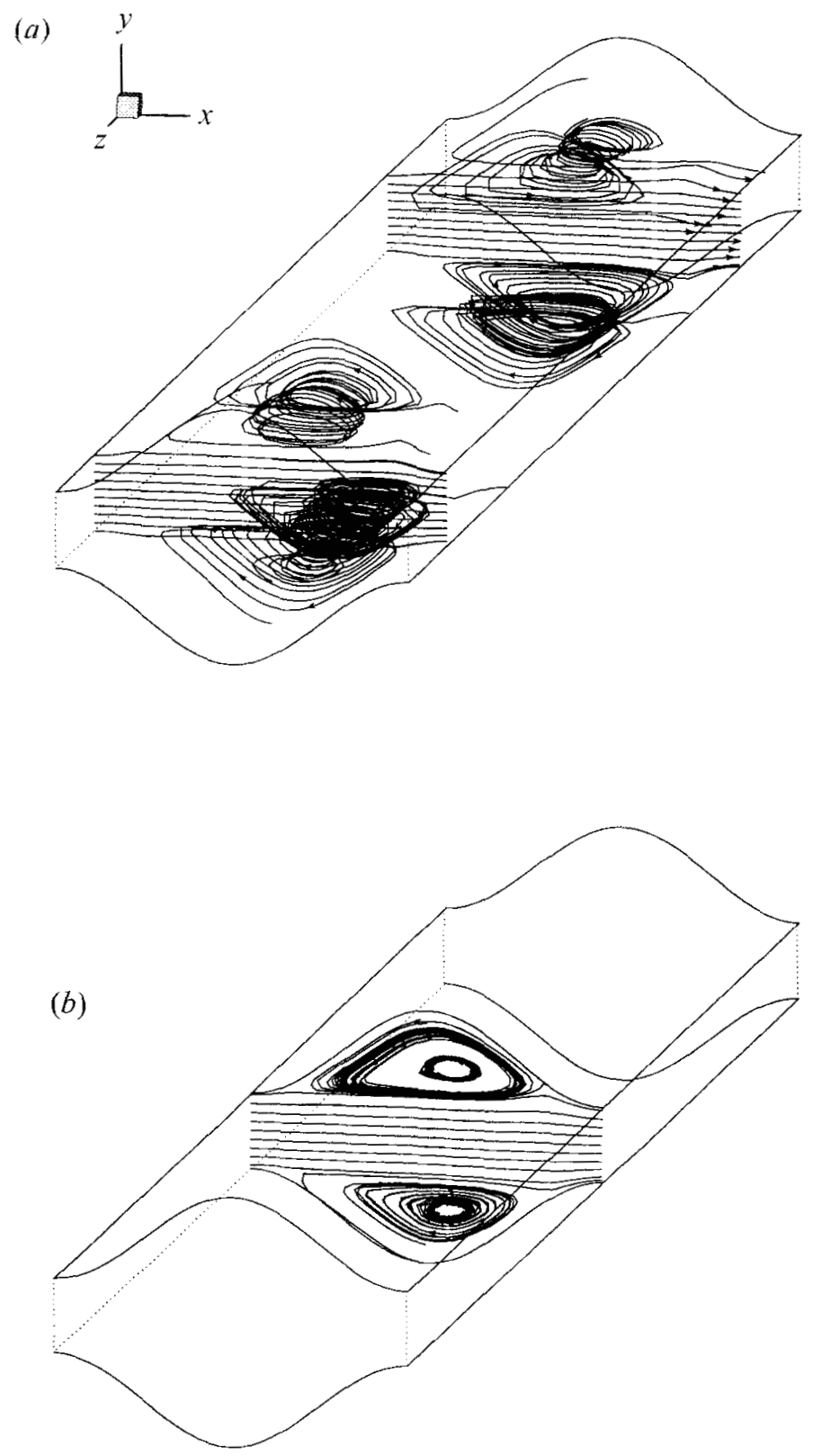

FIGURE 16. Streamtracers of fluid particles located initially at: (a) z-planes near the sidewalls; (b) middle $z$-lane.

$x / h=0$ and an inflectional velocity profile at $x / h=4.67$ along with recirculation flow regions at the upper and lower furrows of the channel. The $y$-axis view, shown in figure $15(c)$, portrays boundary layers at the sidewalls and uniform $U$-velocity profiles along the spanwise direction outside the boundary-layer regions.

Streamtracers of fluid particles initially located at different $z$-planes are shown in figure 16. Fluid particles in the bulk flow convect downstream whereas fluid particles in vortical regions near the sidewalls move towards the centre of the channel until they become entrapped into recirculation zones on z-planes between the initial location and 
the central plane, as shown in figure 16(a). However, streamtracers of fluid particles located at the middle $z$-plane remain on this plane (figure $16 b$ ). Furthermore, their flow patterns consist of recirculation regions which are similar to those obtained from the two-dimensional simulations.

The numerical results of three-dimensional converging-diverging channel flows indicate the presence of stable ordered three-dimensional self-sustained oscillatory flows and the onset of spanwise standing waves at $R e<220$. The velocity time series have demonstrated the existence of a quasi-periodic flow regime with two fundamental frequencies for a Reynolds number of 226. Fourier power spectra calculated in different flow regions indicate that all velocity components display the same fundamental frequencies. To reach this quasi-periodic self-sustained oscillatory state, the flow has undergone two supercritical bifurcations. Our previous two-dimensional simulations have also predicted a quasi-periodic regime at this Reynolds number and two supercritical Hopf bifurcations - the first Hopf bifurcation at $130<R e<150$ and the second at $150<R e<200$ (Guzmán \& Amon 1994a). The primary instability leads to a periodic flow whereas the secondary instability leads to a stable three-dimensional quasi-periodic flow regime with streamwise travelling waves and spanwise standing waves. Thus, the three-dimensional results verify that the converging-diverging open channel flow exhibit a sequence of bifurcations leading to periodic and quasi-periodic flow regimes. Moreover, the spanwise standing waves originating from the secondary instability does not change the early stages of the transition scenario from a steady regime to a quasi-periodic flow by two successive Hopf bifurcations up to Reynoldsnumber flows of 226 .

Time series, velocity profiles, and streamtracer representations from threedimensional simulations have shown that the flow characteristics in the symmetry plane are similar to those obtained with two-dimensional flow assumption simulations. The amplitude of the oscillatory velocity depends on the position of the $z$-plane with respect to the channel sidewalls. Large oscillatory amplitudes are observed at locations near the sidewalls and small amplitudes at points located near the middle plane of symmetry. The effects of the spanwise channel walls, the boundary-layer detachment, and the spanwise standing waves are localized near the sidewalls not affecting the quasi-periodic flow regime and the transition route to chaos for the three-dimensional flows investigated. The possibility of secondary instabilities, induced by threedimensional perturbations at higher Reynolds numbers, remains to be investigated and is currently underway.

\section{Discussion of dynamical flow characterization and future work}

Direct numerical simulations of the governing flow equations enable the characterization of converging diverging channel flows in terms of classical fluid dynamics representations such as instantaneous velocity profiles, velocity time evolutions, streamline fields, and vortical structures. The investigation based on classical fluid dynamics representations has revealed the possibility of triggering self-sustained oscillatory flows in converging-diverging channels and has identified the range of Reynolds numbers for periodic, quasi-periodic and chaotic flow regimes. In addition, we have recognized the source of the instability that induces and sustains these oscillatory flows as being Tollmien-Schlichting instabilities. Furthermore, the classical fluid dynamics characterization demonstrates that the route of transition to turbulence in converging diverging channel flows occurs by successive supercritical Hopf bifurcations, following a scenario similar to that described by the Ruelle- 
Takens-Newhouse theory for onset of chaos. To characterize and quantify weak turbulent and chaotic flow behaviours, we resort to the calculation of dynamical system flow parameters using techniques derived from nonlinear analysis of discrete mechanical systems, such as autocorrelation functions, pseudophase space representations, Eulerian Lyapunov exponents and fractal dimensions.

The dynamical flow characterization of transitional and chaotic regimes enables us to determine the predictability of transitional flows, to characterize chaotic and weak turbulent flows, and to identify flow regions and flow regimes where chaos can be exploited with the objective of improving flow mixing and enhancing heat transfer at a minimum penalty associated with the increase in pumping power. Autocorrelation functions, pseudophase spaces, and Poincaré section representations provide qualitative indications of the unpredictable nature of chaotic flow regimes, of the onset of chaos, and of the topologic structure of the attractors for different flow regimes, whereas Eulerian Lyapunov exponents and fractal dimensions are quantitative tools to establish the onset of chaos, the persistence of chaotic flow regimes, and the long-term persistent unpredictable nature of chaotic flow regimes. In addition, the dynamical flow characterization confirms the evolutive flow pattern and the sequence of bifurcations in the transition to chaos found in converging-diverging channel flows by means of classical fluid dynamics representations.

The predictability of flow regimes is determined by analysing the autocorrelation functions and by representing different attractors in the reconstructed pseudophase spaces. The autocorrelation function results indicate that predictable flow regimes are attained for relatively low supercritical, self-sustained oscillatory flows (e.g. $R e=150$, 400), and unpredictable, aperiodic flow regimes are obtained for higher Reynolds numbers (e.g. $R e=850$ ) beyond the onset of chaos. The transitional flow behaviour is analysed by the geometric visualization of the evolution of the attractors until the appearance of a strange attractor. The pseudophase space and Poincare section representations of the reconstructed attractors reveal that the periodic flow attractor evolves to a well-defined, $T^{2}$ torus, quasi-periodic attractor and, then, to a periodic attractor corresponding to a frequency-locking flow regime as the Reynolds number is increased from 150 to 400 . In this Reynolds number range, the well-organized structure of the flow trajectories confirms the predictability of these flow regimes. The evolution to a strange attractor for higher Reynolds numbers (e.g. $R e=750$ ) from previous quasi-periodic attractors establishes the unpredictable nature of the aperiodic orbits as well as the emergence of a chaotic flow via sequence of successive supercritical bifurcations as described in $\$ 3.4$.

We have first found the frequency-locking phenomenon using classical fluid dynamics representations and, then, verified through dynamical system techniques. This frequency-locking flow regime appears as a consequence of the locking of two fundamental frequencies corresponding to a preceding quasi-periodic regime and evolves to a $T^{3}$ torus as the Reynolds number is increased. The asymptotic plateau reached by the ratio of the fundamental frequencies $\omega_{1} / \omega_{2}$, shown in figure 5 , further confirms the frequency-locking phenomenon. This asymptotic plateau can also be interpreted in the context of structural stability of the frequency-locking phenomenon (Bergé et al. 1986). To analyse the structural stability, the ratio of the fundamental frequencies $\omega_{1} / \omega_{2}$, known also as the winding number $\rho$, is represented as a continuous function of the Reynolds number which is the control parameter. For rational values of $\rho=\omega_{1} / \omega_{2}$, there exists open intervals of the control parameter in which $\rho$ remains constant. The extremes of these intervals correspond to a marginal situation where an infinitesimal change in the Reynolds number leads to a change in the winding number. 
Therefore, the representation of $\rho$ as a continuous function of the control parameter depicts horizontal steps when $\rho$ passes through a rational value and zones where $\rho$ takes continuously changing irrational values.

The pseudophase space representations of different flow regimes portray a dense space of trajectories on the surface of a $T^{2}$ torus for the quasi-periodic flow regime $(R e=250)$ which evolves to a periodic trajectory over another $T^{2}$ torus $(R e=400)$ depicting clearly the frequency-locking phenomenon. The periodic nature of the frequency-locking flow regime is verified by the autocorrelation function, the pseudophase space, and the Poincaré section representation. The evaluation of the Eulerian Lyapunov exponent for this frequency-locking flow regime also verifies its periodic and predictable nature. Moreover, calculations of the largest Eulerian Lyapunov exponent, $\lambda_{E}$, for slightly higher Reynolds numbers, yield nearly vanishing exponents as shown in table 1 . This indicates that the converging-diverging channel flow evolves from the frequency-locking flow regime to a quasi-periodic well-organized flow with three fundamental frequencies before exhibiting any traces representative of chaotic flow behaviour (Guzmán \& Amon 1994a). Frequency-locking flow regimes have also been obtained in other flow systems such as in oscillatory flows around a circular cylinder (Vittori \& Blondeaux 1993); however, these flows exhibit a sudden transition from a phase-locking regime to a chaotic flow for further increases in the control parameter (Gollub \& Benson 1980; Vittori \& Blondeaux 1993), whereas converging-diverging channel flows exhibit multi-frequency quasi-periodic regimes before reaching a chaotic flow regime.

We have found that the converging-diverging channel flow can be represented in low-dimensional phase spaces for Reynolds numbers up to 850 . This assertion of lowdimensional laminar, transitional and weak turbulent converging-diverging flows is confirmed by both the pseudophase space representations of the reconstructed attractors and the calculations of the Eulerian Lyapunov exponents. An embedding dimension of $n=4$ is sufficient to represent the different flow attractors in the pseudophase space since it yields very good agreement with the results previously obtained from classical fluid dynamics characterization such as velocity time series, Fourier power spectra and phase-space portraits. Further indication of the low dimensionality of flow regimes at the onset of chaos is provided by the values of the largest Eulerian Lyapunov exponent, $\lambda_{E}$. The algorithm for calculating $\lambda_{E}$ is sensitive to the embedding dimension $n$ and, after extensive trials, we ascertain that variations no greater than $4 \%$ are obtained in the value of $\lambda_{E}$ by increasing discretely the embedding dimension from $n=3$ to $n=7$ for a wide range of time delays $\tau$ (Guzmán 1995).

The gradual transition to chaos through a sequence of bifurcations is also confirmed by the evolution of the largest Eulerian Lyapunov exponent, $\lambda_{E}$, as a function of the Reynolds number. Vanishing Eulerian Lyapunov exponents correspond to periodic and quasi-periodic flow regimes, whereas, Eulerian Lyapunov exponents greater than zero indicate chaotic flow regimes. The positive value of the largest, long-time average Eulerian Lyapunov exponent, $\lambda_{E}$, indicates the existence of chatic flow regimes for $R e \geqslant 550$. The second largest Eulerian Lyapunov exponent, $\lambda_{2}$, could provide additional information on other directions of exponential growth in the phase space. However, the estimation of $\lambda_{2}$ becomes too resource intensive and computationally expensive because of the increasing amounts of data required to perform the calculations. Nevertheless, we believe that $\lambda_{E}$, reported in this paper with an embedding dimension of four, gives reliable information about the dynamical flow characterization in the range of Reynolds numbers explored, for only minimal 
variations in the value of $\lambda_{E}$ are obtained using higher embedding dimensions. This implies that the flow dynamics in these regimes is captured appropriately by assuming a low-dimensional representation. Consequently, only a small number of degrees of freedom is relevant to investigate the flow dynamics for the onset of chaos and weak turbulent flows in converging-diverging channels.

Low-dimensional, weak turbulence has also been obtained in other flow systems such as in Taylor-Couette flows (Brandstater \& Swinney 1987; Vastano \& Moser 1991), unforced two-dimensional flows past an airfoil (Pulliam \& Vastano 1993), and forced axisymmetric subsonic jets (Broze \& Hussain 1994). We have assumed that converging-diverging flow regimes at the onset of chaos can be represented as lowdimensional systems, and the results of the dynamical flow characterization corroborate this assumption when they are compared with the direct numerical simulations.

High-dimensional chaos has been observed experimentally in flows at relatively large Reynolds numbers, and we expect to appear in numerical simulations of converging-diverging channel flows at higher Reynolds numbers. However, the requirement of data to determine the appropriate dimension of such a system increases dramatically. Consequently, we need to develop a more efficient approach to investigate and achieve a thorough understanding of those complex flow regimes which are required to be represented as high-dimensional dynamical systems. Highdimensional flow behaviours, associated with strong chaotic flow regimes, can be also determined by evaluating the fractal dimension $D_{\lambda}$ given by the Kaplan-Yorke conjecture (Frederickson et al. 1983; Pulliam \& Vastano 1993). The evaluation of $D_{\lambda}$ requires the consideration of other possible directions of exponential growth and the calculation of additional Eulerian Lyapunov exponents. Consequently, the dynamical flow characterization of high-dimensional regimes becomes much more expensive computationally and, in most cases, the computer capability required for DNS that resolves all spatial and temporal scales of motion is beyond the computer power currently available.

The importance of demonstrating, characterizing and quantifying the chaotic and fractal flow behaviours stems from our interest in triggering chaotic flow regimes that induce flow mixing and heat transfer enhancement with small penalty on the power dissipation increase (Amon \& Mikic 1990). With this motivation, the dimension of the strange attractor is a good measure of how well the chaotic orbits fill the phase space and, therefore, is a measure of the chaotic mixing strength. For example, in a twodimensional representation of the attractor in an adequate phase space, a fractal dimension close to two indicates that many stretchings and foldings of fluid elements have occurred, which is indicative of chaos (Ottino et al. 1992). It is in this spirit that we attempt to examine velocity time series using approaches developed originally for nonlinear dynamical system analysis and to link parameters such as fractal dimension and Eulerian Lyapunov exponents with classical flow dynamics characteristics, obtained from DNS of transitional and turbulent flows.

Even though the fractal dimension, $d$, reported in this paper with statistical approach calculations, is not a direct representation of the attractor's fractal geometry, this parameter does give useful information about the future tendency of the velocity evolution and, consequently, about the predictability of flow regimes. An alternative approach we are pursuing is to determine the fractal geometric nature of different attractors by constructing a Poincaré section in an appropriate phase space and, then, computing geometrically the fractal dimension of this section in the phase-space regions. This information may prove useful in evaluating the strength of the attractor 
and its relationship to chaotic flow mixing enhancement in forced convective flows in converging-diverging channels.

\section{Conclusions}

Direct numerical simulations of incompressible, two-dimensional flows in converging-diverging channels reveal the possibility of inducing self-sustained oscillatory flows by triggering and sustaining Tollmien-Schlichting instabilities. This self-sustained oscillatory flow experiences three successive supercritical Hopf bifurcations as the Reynolds number is increased beyond the first flow bifurcation at $130<R e_{c}<135$. Each bifurcation adds an additional frequency while the flow evolves from a laminar to a chaotic flow regime. During this sequence of bifurcations, classical fluid dynamics representations demonstrate the transitional flow evolution from a time-periodic state to a non-periodic broadband flow regime passing through stable non-turbulent regimes of periodicity with one fundamental frequency, quasi-periodicity with two incommensurate fundamental frequencies, frequency-locking periodicity, and quasiperiodicity with three fundamental frequencies and their linear combinations.

Three-dimensional simulations indicate the presence of stable three-dimensional oscillatory flows. The converging-diverging channel flow reaches a quasi-periodic selfsustained oscillatory state at $R e=226$, after two supercritical Hopf bifurcations. The primary instability leads to a periodic flow whereas the secondary instability leads to a stable three-dimensional quasi-periodic flow regime with streamwise travelling waves and spanwise standing waves. Furthermore, the spanwise standing waves originating from the secondary instability have no influence on the early stages of transition to quasi-periodic flows in the range of Reynolds numbers investigated.

The dynamical flow characterization of the reconstructed attractors in threedimensional pseudophase spaces confirms the evolution from a periodic flow attractor to a well-defined $T^{2}$ torus quasi-periodic attractor, to a periodic frequency-locking flow attractor, and to a $T^{3}$ torus which breaks up into a chaotic strange attractor as the Reynolds number is increased from 150 to 500 . The gradual evolution to a strange attractor from previous quasi-periodic regimes establishes the emergence of a chaotic flow behaviour via successive flow bifurcations.

The dynamical characterization of the transitional flow regimes ascertains the Reynolds number range for the onset of chaos. The value of the largest Eulerian Lyapunov exponent verifies that the flow changes its behaviour from a quasi-periodic predictable regime to an aperiodic, chaotic, unpredictable regime in the Reynolds number range between 500 and 550 . For predictable flows in the periodic, quasiperiodic and frequency-locking regimes, the Eulerian Lyapunov exponents are zero, indicating the insensitivity to initial conditions and the exponential decay towards the attractor. For higher Reynolds number flows in the range $550 \leqslant R e \leqslant 850$, the flow remains chaotic, weak turbulent, and low dimensional as verified by the embedding dimension and the almost-constant value of the Eulerian Lyapunov exponents.

The predictable nature of laminar and transitional flow regimes and the unpredictable character of aperiodic flow regimes for $R e>550$ is further verified by the autocorrelation functions, the pseudophase space representations, and the fractal dimension calculations. The chaotic flow behaviour for $R e=850$ is corroborated by the broadband frequency spectrum, by the strange attractor in the pseudophase space, by the asymptotic behaviour to zero of the autocorrelation function, by the positive Eulerian Lyapunov exponent of 1.102538, and by the fractal dimension of 1.376072 indicating the long-term persistent unpredictable nature of this chaotic flow regime. 
Support by the Chilean MIDEPLAN Scholarship, Universidad de Santiago de Chile, Pittsburgh Supercomputer Center, and NSF grant CTS-9311072 is gratefully acknowledged.

\section{REFERENCES}

AMON, C. H. 1993 Spectral element-Fourier method for transitional flows in complex geometries. AIAA J. 31(1), 42.

Amon, C. H. 1995 Spectral element-Fourier method for unsteady conjugate heat transfer in complex geometry flows. AIAA J. Thermophys. Heat Transfer 9(2), 247.

Amon, C. H., Guzmán, A. M. \& Morel, B. 1996 Lagrangian chaos, Eulerian chaos and mixing enhancement in converging-diverging channel flows. Phys. Fluids 8(5), 1192.

Amon, C. H., Herman, C. V., Majumdar, D., Mayinger, F., Mikic, B. B. \& Sekulic, D. 1992 Numerical and experimental studies of oscillatory flows in communicating channels. Intl J. Heat Mass Transfer 35(11), 239.

AMON, C. H. \& Mikic, B. B. 1990 Numerical prediction of convective heat transfer in self-sustained oscillatory flow. AIAA J. Thermophys. Heat Transfer 4, 239.

Amon, C. H. \& Patera, A. T. 1989 Numerical calculation of stable three-dimensional tertiary states in grooved-channel flow. Phys. Fluids A 1, 2005.

Babbiano, A., Boffeta, G., Provenzale, A. \& Vulpiani, A. 1994 Chaotic advection in point vortex models and two-dimensional turbulence. Phys. Fluids 6, 2465.

Barna, G. \& Tsuda, I. 1993 A new method for computing Lyapunov exponents. Phys, Lett. A 175, 421.

Batcho, P. \& Karniadakis, G. E. 1991 Chaotic transport in two- and three-dimensional flow past a cylinder. Phys. Fluids A 3, 1051.

Bergé, P., Pomeau, Y. \& Vidal, C. 1986 Order within Chaos. Wiley, New York.

Brandstater, A. \& Swinney, H. L. 1987 Strange attractors in weakly turbulent Couette--Taylor flow. Phys. Rev. A 35(5), 2207.

BRIGGS, K. 1990 An improved method for estimating Liapunov exponents of chaotic time series. Phys. Lett. A 151, 27.

Broze, G. \& Hussain, F. 1994 Nonlinear dynamics of forced transitional jets: periodic and chaotic attractors. J. Fluid. Mech. 263, 93.

Crisanti, T. J., Falcioni, M., Vulpiani, A. \& Paladin, G. 1991 Lagrangian chaos: transport, mixing and diffusion in fluids. Riv. Nuovo Cimento 14, 1 .

Danielson, T.J. \& Ottino, J. M. 1990 Structural stability in two-dimensional model flows: Lagrangian and Eulerian turbulence. Phys. Fluids A 2, 2024.

Ditto, W. L. \& Pecora, L. M. 1993 Mastering chaos. Sci. Am. 269, 78.

Douglas, J. 1992 Seeking order in chaos. Electric Power Res. Inst. J, 17, 5.

ECKmann, J. P. 1981 Roads to turbulence in dissipative dynamical systems. Rev. Mod. Phys. 53, 643.

Eckmann, J. P., Kamphorst, S. O., Ruelle, D. \& Ciliberto, S. 1986 Liapunov exponents from time series. Phys. Rev. A 34, 4971.

FAGHRI, M. \& Asako, Y. 1987 Numerical determination of heat transfer and pressure drop characteristics for a converging-diverging flow channel. Trans. ASME C: J. Heat Transfer 19, 606.

Feder, J. 1988 Fractal. Plenum Press, New York.

Feigenbaum, M. 1980 The transition to aperiodic behavior in turbulent systems. Commun, Math. Phys. 77, 65.

Frederickson, P., KaPlan, J. A., Yorke, E. D. \& Yorke, J. A. 1983 The Liapunov dimension of strange attractors. J. Diffl Equat. 49, 185.

Gaspard, P. \& Wang, X. J. 1993 Noise, chaos, and (e,t)- entropy per unit time. Phys. Lett. 235(6), 291.

Gollub, J. P. \& Benson, S. H. 1980 Many routes to turbulent convection. J. Fluid Mech. 100, 449. 
Gollub, J. P. \& Swinney, H. L. 1975 Onset of turbulence in a rotating fluid. Phys. Rev. Lett. 35, 927.

Gorman, M., Reith, L. A. \& Swinney, H. L. 1980 Modulation patterns, multiple frequencies and other phenomena in circular Couette flow. Ann. NY Acad. Sci. 357, 10.

Grassberger, P. \& Procaccia, I. 1983 Measuring the strangeness of strange attractors. Physica 9D, 189.

Greiner, M., ChEN, R. F. \& WirTZ, R. A. 1991 Enhanced heat transfer/pressure drop measured from a flat surface in a grooved channel. Trans. ASME C: J. Heat Transfer 113, 498.

GuZMÁN, A. M. 1995 Lagrangian and Eulerian characterization of converging-diverging channel flows: chaos, mixing, and heat transfer enhancement. $\mathrm{PhD}$ thesis, Department of Mechanical Engineering, Carnegie Mellon University.

GuZmán, A. M. \& AMON, C. H. 1993 Flow patterns and forced convective heat transfer in converging-diverging channels. 1993 ASME Heat Transfer Conf. Atlanta, Georgia. In Natural and Forced Convective (ed. M. F. Modest et al.), ASME-HTD, 237, 45.

Guzmán, A. M. \& AmON, C. H. 1994 a Transition to chaos in converging-diverging channel flows: Ruelle-Takens-Newhouse scenario. Phys. Fluids A 6, 1994.

Guzmán, A.M. \& Amon, C.H. $1994 b$ Periodic, quasiperiodic and chaotic regimes in converging-diverging open channel flows. 1994 Intl Mech. Engng Congress and Exposition. In Chaos in Heat Transfer and Fluid Dynamics (ed. V. S. Arpaci et al.), ASME-HTD, 298, 47.

Hattler, B. G., Johnson, P. C., Sawzik, P. J., Shaffer, F. D., Klain, M., Lund, L. W., Reeder, G. D., Walters, F. R., GoOde, J. S. \& Borovetz, H. S. 1992 Respiratory Dialysis: A new Concept in Pulmonary Support. American Society for Artificial Internal Organs Journal 38, 322.

Hurst, H. E. 1951 Long-term storage capacity of reservoirs. Trans. Am. Soc. Civ. Engrs 116, 770.

Keefe, L., MoIn, P. \& KIM, J. 1992 The dimension of attractors underlying periodic turbulent Poiseuille flow. J. Fluid Mech. 242, 1.

LoRenz, E. N. 1963 Deterministic nonperiodic flow. J. Atmos. Sci. 20, 30.

Mclaughlin, J. B. \& OrszaG, S. A. 1982 Transition from periodic to chaotic thermal convection. J. Fluid Mech. 122, 123.

Manneville, P. \& Pomeau, Y. 1980 Different ways to turbulence in dissipative dynamical systems. Physica D 1, 219.

Moon, F. C. 1992 Chaotic and Fractal Dynamics. An Introduction for Applied Scientists and Engineers. Wiley.

Nishimura, T., Murakami, S., Arakawa, S. \& Kawamura, Y. 1990 Flow observations and mass transfer characteristics in symmetrical wavy-walled channels at moderate Reynolds number for steady flow. Intl J. Heat Mass Transfer 33, 835.

Otrino, J. M. 1989 The Kinematics of Mixing: Stretching, Chaos, and Transport. Cambridge University Press.

OTtino, J. M. 1992 New applications of chaos in chemical engineering: intuition versus preduction. In Applied Chaos (ed. J. H. Kim \& J. Stringer). Wiley.

Ottino, J. M., Muzzio, F. J., Tuahjadi, M., Franjone, J. G., Jana, S. C. \& Kusch, H. 1992 Chaos, symmetry, and self-similarity: exploiting order and disorder in mixing processes. Science 257, 754 .

Packard, N. H., Crutchfield, J. P., Farmer, J. D. \& Shaw, R. S. 1980 Geometry from a time series. Phys. Rev. Lett. 45, 712.

PAterA, A. T. 1984 A spectral element method for fluid dynamics: laminar flow in a channel expansion. J. Comput. Phys. 54, 468.

Pfister, G., Buzug, T. \& ENGe, N. 1992 Characterization of experimental time series from Taylor-Couette flow. Physica D 58, 441.

Pulliam, T. H. \& Vastano, J. A. 1993 Transition to chaos in an open unforced 2D flow. J. Comput. Phys. 105, 133.

RaLPH. M. E. 1986 Oscillatory flows in wavy-walled tubes. J. Fluid Mech. 168, 515.

RaYleigh, J. W. S. 1945 The Theory of Sound, reprint of 2nd edn. Dover, New York.

Rom-KeDAR, V., LEONARD, A. \& Wiggins, S. 1990 An analytical study of transport, mixing, and chaos in an unsteady vortical flow. J. Fluid Mech. 214, 347. 
Rosenstein, M., Collins, J. J. \& De Luca, C. J. 1993 A practical method for calculating largest Lyapunov exponents from small data sets. Physica D 65, 117.

Ruelle, D. 1980 Strange attractors. The Mathematical Intelligencer 2, 126.

Ruelle, D. 1992 Chaotic Evolution and Strange Attractors. Cambridge University Press.

Ruelle, D. \& Takens, F. 1971 On the nature of turbulence. Commun. Math. Phys. 20, 167.

SCHATZ, M. F. \& SwINNEY, H. L. 1992 Secondary instability in plane channel flow with spatially periodic perturbations. Phys. Rev. Lett. 69, 434.

Schepers, H. E., van Beck, J. H. G. M. \& Bassingthwaighte, J. B. 1992 Four methods to estimate the fractal dimension from self-affine signals. IEEE J. Engng Medicine Biol. 57.

Shinbrot, T., Grebogi, C., OTt, E. \& Yorke, J. A. 1993 Using small perturbations to control chaos. Nature 363, 441 .

SoBEy, I. J. 1980 On flow through furrowed channels. Part 1. Calculated flow patterns. J. Fluid Mech. 96, 1.

SOBEY, I. J. 1982 Oscillatory flow at intermediate Strouhal number in asymmetric channels. J. Fluid Mech. 125, 359.

SoBEY, I. J. 1985 Dispersion caused by separation during oscillatory flow through a furrowed channel. Chem. Engng Sci. 40, 2129.

Sparrow, E. M. \& Prata, A. T. 1983 Numerical solutions for laminar flow and heat transfer in a periodically converging diverging tube with experimental confirmation. Numer. Heat Transfer 6, 441 .

Stephanoff, K. D., Sobey, I. J. \& Bellhouse, B. J. 1980 On flow through furrowed channels. Part 2. Observed flow patterns. J. Fluid Mech. 96, 27.

TAKENS, F. 1981 Detecting strange attractors in turbulence. In Dynamical Systems and Turbulence, Warwick 1980. Lecture Notes in Mathematics, vol. 898, p. 336, Springer.

Vastano, J. A. \& Moser, R., D. 1991 Short-time Lyapunov exponent analysis and the transition to chaos in Taylor-Couette flow. J. Fluid Mech. 223, 83.

VitTori, G. \& Blondeaux, P. 1993 Quasiperiodicity and phase locking route to chaos in the 2-D oscillatory flow around a circular cylinder. Phys. Fluids A 5, 1866.

Walden, R. W. Kolodner, P., PAssner, A. \& Surko, C. M. 1984 Nonchaotic Rayleigh-Bénard convection with four and five incommensurate frequencies. Phys. Rev. Lett. 53, 242.

Wolf, A., Swift, J. B., Swinney, H. L. \& Vastano, J. A. 1985 Determining Lyapunov exponents from a time series. Physica D 16, 285. 